\title{
Patterns of resource use by fishes and macroinvertebrates in Barataria Bay, Louisiana
}

\author{
R. F. Jones ${ }^{1, *}$, D. M. Baltz ${ }^{1,2, * *}$, R. L. Allen ${ }^{2}$ \\ ${ }^{1}$ Department of Oceanography and Coastal Sciences, and ${ }^{2}$ Coastal Fisheries Institute, Louisiana State University, \\ Baton Rouge, Louisiana 70803-7503, USA
}

\begin{abstract}
We examined patterns of resource use by size classes of fishes and macroinvertebrates in Barataria Bay, Louisiana. Analyses were based on microhabitat data characterized by water depth, distance from shore, substrate, salinity, dissolved oxygen and temperature. Stratified random samples along the salinity gradient from the nearshore Gulf of Mexico to about $30 \mathrm{~km}$ inland were taken monthly with a $1 \mathrm{~m}$ beam trawl to characterize distributions of fishes, macroinvertebrates and environmental conditions. From October 1992 through September 1993, 31602 individuals belonging to 70 species were collected. The 10 most abundant species plus 3 others of special interest were chosen for further analyses. Seasonal differences in environmental variables and differences in resource use among species within ecological groups and within selected species were evaluated by 1 -way ANOVAs. A factor analysis resolved 6 environmental variables into 3 orthogonal axes that simplified visualization and comparisons within and among species. Three factors accounted for $70 \%$ of the environmental variance and represented seasonality, distance-depth and substrate-salinity axes. Twelve of 13 species showed statistically significant differences between size classes in use of temperature, but some differences were probably due to seasonal temperature changes and the ephemeral nature of life history stages. Within-species differences were less prevalent for variables other than temperature, and the majority were related to use of deeper water as size increased. When seasonal, spatial and size-structured distributions were compared, species with peak abundances during the same seasons had the highest ecological overlaps. Ecological overlaps of 3 closely related species pairs were generally below the mean for the assemblage. Understanding patterns of resource use and the environmental requirements of estuarine-dependent species is an important step in identifying and protecting nursery habitats.
\end{abstract}

KEY WORDS: Beam trawl $\cdot$ Estuary $\cdot$ Juveniles $\cdot$ Microhabitat · Ontogeny $\cdot$ Recruitment

Resale or republication not permitted without written consent of the publisher

\section{INTRODUCTION}

Saltmarsh estuaries are presumed to enhance the growth and survival of individuals that find more favorable conditions within estuaries than in other nearby coastal habitat types (Boesch \& Turner 1984). Understanding habitat selection of early life history stages of fishes and macroinvertebrates provides insights into variables that control their survival and

\footnotetext{
*Present address: Northwest Indian Fisheries Commission, PO Box 1029, Forks, Washington 98331, USA

${ }^{* *}$ Corresponding author. E-mail: dbaltz@lsu.edu
}

growth by providing appropriate physiological conditions, adequate prey and reduced predation. Egg and larval stages have the highest and most variable mortality rates, and success at this stage can often be correlated with recruitment. Nevertheless, recruitment success can also depend on successfully clearing a series of obstacles throughout an extensive life history (Walters \& Collie 1988), and poor recruitment may be the result of just 1 ecological obstacle (i.e. a bottleneck, sensu Wiens 1977).

As individual size increases during development, ecological requirements change, cohort numbers decline and opportunities for compensatory mortality 
diminish. Mortality can be highly compensatory in fishery populations, especially in the earliest life history stages, and high levels of early mortality may not have a significant effect on adult population levels (Houde 1989, Rose et al. 1993). Nevertheless, chronic or episodic mortality can potentially have a greater impact on recruitment if it occurs at or shortly after a spawning peak or in a discreet nursery area, e.g. an estuary. As the larvae of estuarine-dependent species make the transition to juveniles and select nursery habitats, their survival (Minello et al. 1989) and growth (Baltz et al. 1998) rates are sensitive to environmental conditions, which can have effects similar to those hypothesized for larval stages (Houde 1989). Either poor quality or a reduced quantity of suitable habitat within estuaries may restrict recruitment opportunities for some estuarine-dependent populations by reducing growth or increasing predation risks for juveniles (Beck 1995). Variability in recruitment may be controlled by many complex interactions between organisms and their environments (Miller 1994). Both density-dependent and stochastic causes can work simultaneously on recruitment for a given population (Jobling 1995), but without the appropriate environmental conditions for a life history stage, there is limited opportunity for density-dependent effects to influence recruitment to the next life history stage. The focus of research in recruitment has changed from stock-recruitment models to studies of underlying mechanisms and processes that control year-to-year recruitment variability (Houde 1987, Miller et al. 1988, 1991, Miller 1994). Variables including the effects of adult condition on breeding success, effects of food supply on growth and survival of larvae, predation effects on early life stages, abiotic variables, quantity and quality of habitat and the effects of latitude and climate change on these variables are often examined as possible causes of variability in recruitment (Hjort 1914, Cushing 1973, Miller 1994, Jobling 1995).

Establishing the habitat requirements of juvenile estuarine-dependent fishes and macroinvertebrates is a vital initial step toward understanding the relative importance of abiotic environmental variables in conjunction with other variables that may control recruitment (Allen \& Baltz 1997, Baltz et al. 1998). Overfishing, pollution and habitat alteration have led to recent declines in catches of estuarine-dependent species in many areas (Houde \& Rutherford 1993); however, commercial catches in Louisiana have not declined significantly (Chesney et al. 2000). In Louisiana, subsidence, eustatic sea-level rise, along with saltwater intrusion and lack of sediment input, have been responsible for an annual loss of wetlands of up to $65 \mathrm{~km}^{2} \mathrm{yr}^{-1}$ (Day et al. 1989, Turner 1997); however, other habitat changes are currently either offsetting or confounding anti- cipated effects of marsh loss (Chesney et al. 2000, Chesney \& Baltz 2001) and recruitment rates for some shrimp species appear to be increasing (Zimmerman et al. 2000).

Our purpose here is to examine microhabitat use patterns by characterizing sites selected by individuals to describe population responses to spatial and temporal environmental gradients. Our focus was on juveniles of estuarine-dependent species that are capable of habitat selection. For aquatic vertebrates, abiotic variables may be usefully viewed as ecological resources (Magnuson et al. 1979, Hurlbert 1981) when we describe the niche as the set of resources used by an organism (i.e. energy, materials and site). The abiotic characteristics of sites occupied by individuals may include materials that are actually consumed (oxygen), that are used to build nests (substrate particles), or that constrain or modify utilization of other resources (salinity or temperature). At the finest scale, the microhabitat of an individual is the site it occupies at a given point in time, but when individuals cannot be directly observed, coarser measurements are usually necessary (Hurlbert 1981, Baltz et al. 1993). Presumably, fishes and macroinvertebrates select a site to occupy in response to variables that optimize their net energy gain while avoiding predators and competitors. Since individuals of similar size within a species select similar microhabitats, independent measurements of many individuals and the associated physical, chemical and biological variables can be used to define size class and population responses to environmental gradients (Baltz 1990). Based on an analysis of microhabitat data, we examined abiotic resource use at the species level and patterns of ontogenetic change in use among size classes of selected fishes and macroinvertebrates to answer 2 questions: (1) Does segregation occur among members of important ecological groups? (2) Do dominant species show discernable ontogenetic shifts, and can patterns of use be illustrated to facilitate the development of essential fish habitat criteria for habitat restoration and management?

\section{MATERIALS AND METHODS}

Study area. Barataria Basin, including Barataria Bay and Caminada Bay, is about $50 \mathrm{~km}$ wide where it meets the Gulf of Mexico and extends northerly about $110 \mathrm{~km}$ along a salinity gradient ending in freshwater marshes (Conner \& Day 1987). It lies between the natural levees of the Mississippi River and the abandoned Bayou Lafourche distributary (Fig. 1). The basin has been closed to riverine input of sediment and fresh water since the Bayou Lafourche-Mississippi River connection was cut off in 1902 and the construction of 


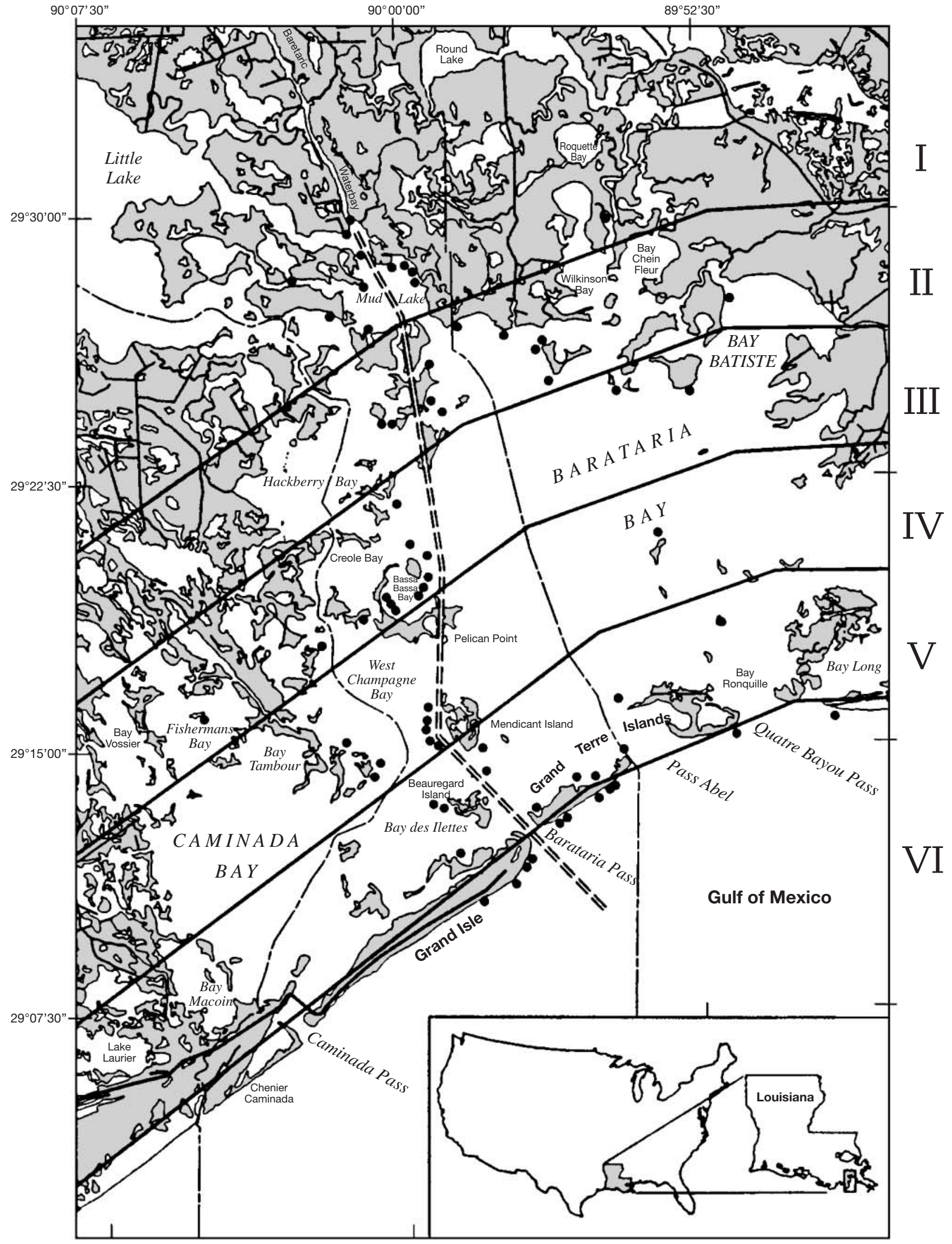

Fig. 1. Barataria Basin study area with dots representing beam-trawl locations in 6 strata (I to VI) 
artificial levees completed in the 1930s to 1940s (Conner \& Day 1987). The absence of sediment input, along with subsidence, canal construction for petroleum production, drainage and navigation, and saltwater intrusion, have been responsible for extensive wetland losses in the basin (Thompson \& Forman 1987, Day et al. 1989). Today the primary source of fresh water for the basin is precipitation, which averages $160 \mathrm{~cm} \mathrm{yr}^{-1}$ (Baumann 1987).

The saltmarsh area of the basin totals about 145000 ha with open water accounting for more than 82000 ha of that total (Conner \& Day 1987). The dominant vegetation is smooth cordgrass Spartina alterniflora, which accounts for $63 \%$ of plant coverage (Conner et al. 1987); other species include black rush Juncus roemarianus and saltgrass Distichilis spicata. The water is shallow, usually $<2 \mathrm{~m}$ deep, turbid and salinity varies seasonally and spatially from about 6 to $22 \mathrm{psu}$ in the saltmarsh portion of the study area (Baltz et al. 1993, Allen \& Baltz 1997). The microtidal system, with a range of $0.32 \mathrm{~m}$, is dominated by seasonal winds usually associated with the passage of cold fronts (Childers et al. 1990).

Field methods. From October 1992 through September 1993, 373 beam-trawl samples were collected from 72 different locations in Barataria Basin and the adjacent nearshore Gulf of Mexico out to about $2 \mathrm{~km}$ from shore. All samples were collected in conjunction with an earlier study on flatfishes (Allen \& Baltz 1997). From the preserved specimens, we analyzed all fishes and nektonic macroinvertebrates. Juveniles were collected quantitatively in Barataria Basin using a $1 \mathrm{~m}$ beam trawl with a uniform mesh size of $5 \mathrm{~mm}$ (Kuipers 1975). To aid sampling on fine substrates, Kuipers' design was modified by adding wider polyvinyl chloride skids $(10 \times 50 \mathrm{~cm})$.

Sampling effort in the lower portion of the broad saline-to-brackish estuary was distributed evenly over 6 strata (Fig. 1) along the salinity gradient from the nearshore Gulf of Mexico to the brackish marsh about $30 \mathrm{~km}$ inland. Sampling at locations within strata involved a series of tows (usually 5) at sites parallel to the shoreline, usually the marsh edge. Each tow was further from shore and usually in deeper water than the previous one. Tow duration at each site was short (range 1 to $2 \mathrm{~min}$ ), and tows were made parallel to the shoreline to minimize heterogeneity of environmental conditions within samples. Distance trawled varied with wind, current, sea state and substrate type. Each 2 min tow covered a mean $( \pm 1 \mathrm{SD})$ area of approximately $152 \pm 14.8 \mathrm{~m}^{2}$ (Allen \& Baltz 1997). Temperature $\left({ }^{\circ} \mathrm{C}\right)$, salinity (psu) and dissolved oxygen $\left(\mathrm{mg} \mathrm{l}^{-1}\right)$ were measured in the water column at each tow site with YSI Dissolved Oxygen and SCT meters. Substrate type was characterized as dominant and subdominant components from surface samples taken along each tow site and coded on an ordinal scale of particle size: $1=$ clay, $2=$ silt, $3=$ sand, $4=$ plant detritus (primarily Spartina alterniflora) and $5=$ shell and shell fragments. Water depth (m) was recorded at the beginning and end of each tow and depth was estimated as the mean of the 2. Distance from shore (m) was also estimated as the nearest point during each tow.

Trawl samples were sorted in the field to separate all fishes and macroinvertebrates. Specimens were preserved in $95 \%$ ethanol. All fishes were identified in the laboratory with descriptions and keys by Hoese \& Moore (1977), Martin \& Drewry (1978), Fahay (1983), Munroe (1991) and Munroe et al. (2000) and were counted and measured to the nearest $\mathrm{mm}$ total length (TL). Macroinvertebrates, excluding most mollusks, were identified using descriptions and keys by Hopkins et al. (1989) and Williams (1984), and were counted and measured to the nearest $\mathrm{mm}$ TL except for blue crab, lesser blue crab and Florida lady crab, which were measured to the nearest $\mathrm{mm}$ carapace width (CW).

Analytical methods. Selected fishes and macroinvertebrates were assigned to 4 size classes (SC1 to SC4: $\mathrm{SC} 1 \leq 15 \mathrm{~mm}, 15 \mathrm{~mm}<\mathrm{SC} 2 \leq 30 \mathrm{~mm}, 30 \mathrm{~mm}<\mathrm{SC} 3 \leq$ $100 \mathrm{~mm}$, and SC4 > $100 \mathrm{~mm}$ TL or $\mathrm{CW}$ ) to evaluate size-related patterns of resource use (Livingston 1988). Each beam-trawl collection was treated as an independent sample to calculate univariate means and SEs of environmental variables and densities of species and size classes. Since the abundances of individuals varied considerably among samples, the environmental means used by species or size classes were weighted to estimate population responses to environmental gradients without increasing degrees of freedom (Hurlbert 1984). Transformed environmental variables $(\log x+1)$, the General Linear Model (GLM) procedure and the least-square-mean (LSM) comparisons (SAS Institute 1996) were used in univariate analyses of variance (ANOVAs) to test weighted means of environmental variables among seasons and within and among selected species. Four seasons were defined as winter (December to February), spring (March to May), summer (June to August) and fall (September to November). Distance from shore was included in the analysis to assess uniformity in the sampling regime across seasons. For among-species comparisons, 1 record per species present in each sample was generated and the analysis weighted by relative abundance (number of individuals caught per min). A similar data set was also generated to examine resource-use patterns of size classes within species. Interactions of seasonal effects and size classes were excluded from the analytical design because the ephemeral occurrence of many size classes yielded adequate sample sizes in only 1 or 
2 seasons. Size classes that were poorly characterized in the samples were excluded from ANOVA.

We also calculated overlaps (Schoener 1970) in temporal, spatial and size-structured (Livingston 1988) distributions between species to assess resource partitioning (Ross 1986) across 4 seasons, 6 strata and 4 size classes. Proportional overlaps in relative abundances (based on catch per min) were used to estimate overlap between selected species pairs in arrays of seasons, strata and size classes $(4 \times 6 \times 4)$ as well as overlap between species pairs by season and strata without regard to size.

To understand and visualize changing community structure across important environmental axes, we examined variation in resource use patterns in a principal component analysis of 6 environmental variables. The analysis was based on the correlation matrix and the first 3 principal components were rotated using the Varimax option in the SAS Factor Procedure (SAS Institute 1996). This factor analysis resolved 6 intercorrelated microhabitat variables into 3 orthogonal factors that simplified visual representation and comparisons within and among species. From 373 samples without missing data, environmental variables were used to calculate variable loadings and generate factor scores for each sample. The factor scores for each sample were then used to calculate centroids for each species and size class. The relative abundance of the species or size class was used to weight the calculation of centroids to reflect the population's numerical responses to each factor.

\section{RESULTS}

\section{Environmental variation}

A wide range of environmental conditions was sampled over the course of the year. Water depth ranged from 0.5 to $6.5 \mathrm{~m}$ while distance from shore ranged from 5 to $3660 \mathrm{~m}$. Salinity ranged from 0.9 to $29.2 \mathrm{psu}$ throughout the study area and varied both temporally and spatially. Temperature and dissolved oxygen also varied temporally and spatially from 10.2 to $32.0^{\circ} \mathrm{C}$ and 4.4 to $12.1 \mathrm{mg} \mathrm{l}^{-1}$. Substrate type ranged from clay to shell fragments (and whole shells) and often varied among the 5 tow sites at a single location. Organic detritus was common near the marsh edge, but as distance from shore increased, detritus decreased and fine-grain particles were dominant (i.e. clay and silt). Shells were prevalent in many trawls and ranged in size from small fragments of a few $\mathrm{mm}$ to live oysters Crassostrea virginica that were greater than $100 \mathrm{~mm}$ in their longest dimension.

There was considerable seasonal variation of environmental conditions, characterized by stratified random sampling (Table 1). At least 1 seasonal difference was significant for depth, substrate, salinity, dissolved oxygen and temperature (1-way ANOVAs: $F$-ratio $\geq 4.40, \mathrm{df}=3$ and 369, $\mathrm{p} \leq 0.005)$. Distance from shore was the only variable that did not differ and reflected uniformity in the sampling regime across seasons. Mean depths sampled in winter were significantly lower than depths sampled in spring or fall, but were similar to depths sampled in summer. Mean substrate particle size was significantly higher in winter than in spring or summer, but was not significantly different from the fall mean. Salinity was highest in the fall and spring and lowest in the summer and winter. Salinity differences along the northsouth gradient were also greatest in the fall with a range of 17 psu. Means of dissolved oxygen concentrations measured during daylight hours were significantly higher in the winter, intermediate in spring and fall (but not detectably different) and significantly lower in the summer. Mean water temperatures differed significantly over all seasons. Summer water temperatures were higher than fall, which were higher than spring, and winter mean water temperatures were the lowest.

Table 1. Seasonal means ( $\pm 1 \mathrm{SE}$ ) for environmental variables measured at 373 beam-trawl sites in Barataria Basin, Louisiana, between October 1992 and September 1993. See text for substrate (particle size) codes. Posterior testing after a finding of overall significant differences ( $F$-ratio $\geq 4.40, \mathrm{df}=3$ and $369, \mathrm{p} \leq 0.005$ ) among seasonal means for each environmental variable is based on LSM comparisons. Differences among seasonal means are denoted by different letters (i.e. A, B, C and D) reading horizontally

\begin{tabular}{|lcccc|}
\hline Variable & Dec to Feb & Mar to May & Jun to Aug & Sep to Nov \\
\hline Depth $(\mathrm{m})$ & $1.6 \pm 0.05 \mathrm{~A}$ & $1.9 \pm 0.08 \mathrm{BC}$ & $1.7 \pm 0.09 \mathrm{AB}$ & $1.9 \pm 0.07 \mathrm{C}$ \\
Distance $(\mathrm{m})$ & $294 \pm 41.2$ & $230 \pm 27.2$ & $226 \pm 36.1$ & $288 \pm 42.1$ \\
Substrate code & $2.5 \pm 0.1 \mathrm{~A}$ & $2.0 \pm 0.1 \mathrm{~B}$ & $1.8 \pm 0.1 \mathrm{~B}$ & $2.1 \pm 0.1 \mathrm{AB}$ \\
Salinity $(\mathrm{psu})$ & $9.8 \pm 0.50 \mathrm{~A}$ & $13.9 \pm 0.70 \mathrm{~B}$ & $11.0 \pm 0.64 \mathrm{~A}$ & $15.1 \pm 0.89 \mathrm{~B}$ \\
Dissolved oxygen $\left(\mathrm{mg} \mathrm{l}^{-1}\right)$ & $9.3 \pm 0.09 \mathrm{~A}$ & $8.1 \pm 0.14 \mathrm{~B}$ & $7.6 \pm 0.13 \mathrm{C}$ & $8.2 \pm 0.17 \mathrm{~B}$ \\
Temperature $\left({ }^{\circ} \mathrm{C}\right)$ & $15.7 \pm 0.19 \mathrm{~A}$ & $19.9 \pm 0.54 \mathrm{~B}$ & $30.1 \pm 0.13 \mathrm{C}$ & $23.5 \pm 0.47 \mathrm{D}$ \\
No. of tows & 120 & 85 & 80 & 88 \\
\end{tabular}


Table 2. Species of fishes and macroinvertebrates listed in order of numerical abundance from 373 beam-trawl samples collected in Barataria Basin, Louisiana, between October 1992 and September 1993

\begin{tabular}{|c|c|c|c|c|}
\hline Common name & Scientific name & otal no. caught & Total caught (\%) & Frequency of occurrence \\
\hline Bay anchovy & Anchoa mitchilli & 8833 & 28.0 & 68.4 \\
\hline Atlantic croaker & Micropogonias undulatus & 4327 & 13.7 & 54.9 \\
\hline Blue crab & Callinectes sapidus & 3717 & 11.8 & 72.7 \\
\hline Brown shrimp & Farfantepenaeus aztecus & 2538 & 8.0 & 53.3 \\
\hline Lesser blue crab & Callinectes similis & 2085 & 6.6 & 39.0 \\
\hline Darter goby & Gobionellus boleosoma & 1839 & 5.8 & 44.0 \\
\hline Bay whiff & Citharichthys spilopterus & 1583 & 5.0 & 60.7 \\
\hline Offshore tonguefish & Symphurus civitatum & 1512 & 4.8 & 40.3 \\
\hline Roughneck shrimp & Trachypenaeus similis & 570 & 1.8 & 10.3 \\
\hline Fringed flounder & Etropus crossotus & 483 & 1.5 & 38.5 \\
\hline Spot & Leiostomus xanthurus & 452 & 1.4 & 15.1 \\
\hline Grass shrimp & Palaeomonetes pugio & 439 & 1.4 & 19.4 \\
\hline Least puffer & Sphoeroides parvus & 396 & 1.3 & 23.9 \\
\hline Naked goby & Gobiosoma bosc & 392 & 1.2 & 16.4 \\
\hline Blackcheek tonguefish & Symphurus plagiusa & 322 & 1.0 & 34.7 \\
\hline Sea catfish & Arius felis & 271 & 0.9 & 16.2 \\
\hline White shrimp & Litopenaeus setiferus & 268 & 0.8 & 29.7 \\
\hline Sand seatrout & Cynoscion arenarius & 239 & 0.8 & 17.8 \\
\hline Gulf menhaden & Brevoortia patronus & 168 & 0.5 & 12.7 \\
\hline Southern kingfish & Menticirrhus americanus & 155 & 0.5 & 13.8 \\
\hline Seabob & Xiphopenaeus kroyeri & 124 & 0.4 & 4.5 \\
\hline Bighead searobin & Prionotus tribulus & 117 & 0.4 & 15.1 \\
\hline Bank cusk eel & Ophidion holbrooki & 85 & 0.3 & 5.3 \\
\hline Green goby & Microgobius thalassinus & 68 & 0.2 & 11.7 \\
\hline Florida lady crab & Ovalipes floridanus & 59 & 0.2 & 4.8 \\
\hline Chain pipefish & Syngnathus louisianae & 54 & 0.2 & 11.1 \\
\hline Inshore lizardfish & Synodus foetens & 53 & 0.2 & 8.5 \\
\hline Skilletfish & Gobiesox strumosus & 53 & 0.2 & 5.6 \\
\hline Speckled worm eel & Myrophis punctatus & 50 & 0.2 & 11.4 \\
\hline Sharptail goby & Gobionellus hastatus & 45 & 0.1 & 11.9 \\
\hline Southern flounder & Paralichthys lethostigma & 45 & 0.1 & 6.9 \\
\hline Atlantic brief squid & Lolliguncula brevis & 30 & $<0.1$ & 3.2 \\
\hline Spotted seatrout & Cynoscion nebulosus & 29 & $<0.1$ & 3.7 \\
\hline Lined sole & Achirus lineatus & 28 & $<0.1$ & 5.6 \\
\hline Atlantic spadefish & Chaetodipterus faber & 17 & $<0.1$ & 3.4 \\
\hline Gulf butterfish & Peprilus burti & 17 & $<0.1$ & 2.4 \\
\hline Banded drum & Larimus fasciatus & 16 & $<0.1$ & 3.2 \\
\hline Southern hake & Urophycis floridana & 15 & $<0.1$ & 2.4 \\
\hline Lesser rock shrimp & Sicyonia dorsalis & 15 & $<0.1$ & 0.8 \\
\hline Atlantic bumper & Chloroscombrus chrysurus & 11 & $<0.1$ & 1.3 \\
\hline Hogchoker & Trinectes maculatus & 10 & $<0.1$ & 2.7 \\
\hline Pinfish & Lagodon rhomboides & 9 & $<0.1$ & 2.1 \\
\hline Striped anchovy & Anchoa hepsetus & 8 & $<0.1$ & 2.1 \\
\hline Atlantic stingray & Dasyatis sabina & 7 & $<0.1$ & 1.9 \\
\hline Planehead filefish & Monacanthus hispidus & 6 & $<0.1$ & 1.3 \\
\hline Skipjack herring & Alosa chrysochloris & 5 & $<0.1$ & 0.3 \\
\hline Inland silverside & Menidia beryllina & 3 & $<0.1$ & 0.8 \\
\hline Sheepshead & Archosargus probatocephalus & 3 & $<0.1$ & 0.8 \\
\hline Star drum & Stellifer lanceolatus & 3 & $<0.1$ & 0.8 \\
\hline Violet goby & Gobioides broussoneti & 3 & $<0.1$ & 0.8 \\
\hline Silver perch & Bairdiella chrysoura & 3 & $<0.1$ & 0.5 \\
\hline Crevalle jack & Caranx hippos & 2 & $<0.1$ & 0.5 \\
\hline Spotfin mojarra & Eucinostomus argenteus & 2 & $<0.1$ & 0.5 \\
\hline Gulf toadfish & Opsanus beta & 2 & $<0.1$ & 0.3 \\
\hline Southern puffer & Sphoeroides nephelus & 1 & $<0.1$ & 0.3 \\
\hline Rough silverside & Membras martinica & 1 & $<0.1$ & 0.3 \\
\hline Ladyfish & Elops saurus & 1 & $<0.1$ & 0.3 \\
\hline Gray snapper & Lutjanus griseus & 1 & $<0.1$ & 0.3 \\
\hline Red drum & Sciaenops ocellatus & 1 & $<0.1$ & 0.3 \\
\hline Pink shrimp & Penaeus duorarum & 1 & $<0.1$ & 0.3 \\
\hline
\end{tabular}


Table 2 (continued)

\begin{tabular}{|llccc|}
\hline Common name & Scientific name & Total no. caught & Total caught (\%) & Frequency of occurrence \\
\hline Gulf pipefish & Syngnathus scovelli & 1 & $<0.1$ & 0.3 \\
Lookdown & Selene vomer & 1 & $<0.1$ & 0.3 \\
Southern stargazer & Astroscopus y-graecum & 1 & $<0.1$ & 0.3 \\
Freckled blenny & Hypsoblennius ionthas & 1 & $<0.1$ & 0.3 \\
Crested cusk eel & Ophidion welshi & 1 & $<0.1$ & 0.3 \\
Emerald sleeper & Erotelis smaragdus & 1 & $<0.1$ & 0.3 \\
Code goby & Gobiosoma robustum & 1 & $<0.1$ & 0.3 \\
Sand perch & Diplectrum formosum & 1 & $<0.1$ & 0.3 \\
Feather blenny & Hypsoblennius hentz & 1 & $<0.1$ & 0.3 \\
Lancer stargazer & Kathetostoma albigutta & 1 & & 0.3 \\
Total number & & 31602 & & \\
\hline
\end{tabular}

\section{Assemblage structure}

In 373 beam-trawl samples, 31602 individuals belonging to 70 species were identified (Table 2). Bay anchovy was the dominant species overall constituting over a quarter of the total catch by number, while blue crab was the dominant macroinvertebrate species accounting for about $12 \%$ of the total catch. The 10 most abundant species included fishes $(58.8 \%$ by number), crabs $(18.4 \%)$ and shrimp (9.8\%), and to- gether accounted for $87 \%$ of the total catch. Three additional species, spot, blackcheek tonguefish and white shrimp, were also included for comparisons between closely related species, and were abundant enough to permit valid statistical analyses. Collectively, the 13 species selected amounted to more than $90 \%$ of the total catch.

For the selected species, most individuals fell within $\mathrm{SC} 3$ (>30 to $100 \mathrm{~mm}$ ), but sizes ranged from transforming juveniles (5 to $10 \mathrm{~mm}$ ) to adults (Figs. $2,3 \& 4$ ). The
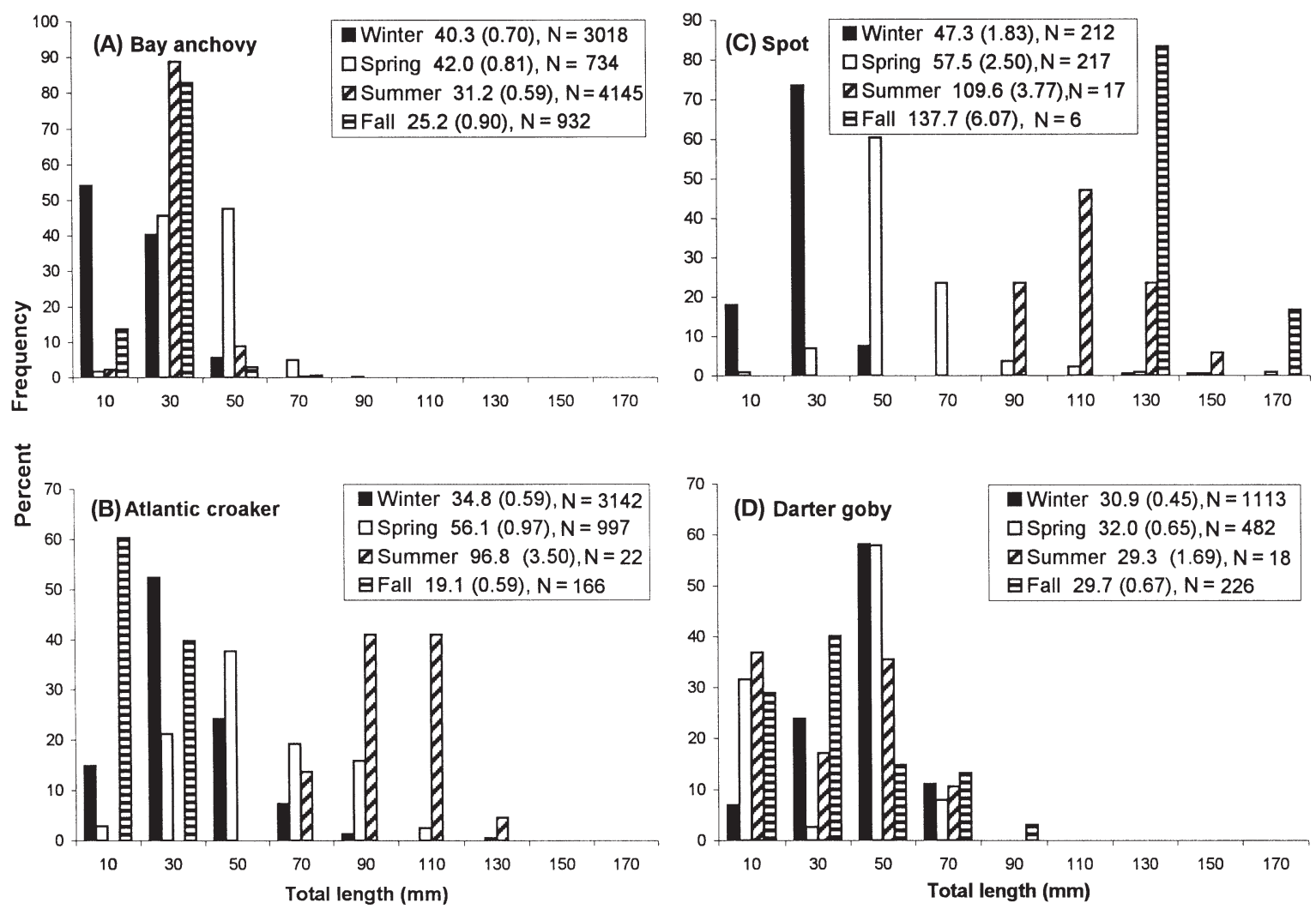

Fig. 2. Seasonal size frequency distributions for 4 common water column fishes in the Barataria Basin: (A) bay anchovy, (B) Atlantic croaker, (C) spot and (D) darter goby. Numbers in legends are mean lengths (SE); $\mathrm{N}=$ total number of individuals 
Table 3. Weighted means \pm 1 SE for environmental variables used by 13 selected fish and invertebrate species in Barataria Bay, Louisiana. $\mathrm{N}$ is the number of independent samples, while total number is the number of individuals. Following overall significant differences among species means ( $F$-ratio $\geq 5.48, \mathrm{df} \geq 3$ and $878, \mathrm{p} \leq 0.001$ ) within 3 ecological groups, posterior testing based on LSM comparisons ( $\mathrm{p} \leq 0.05$ ) is indicated by different letters (i.e. A, B, C and D) reading vertically

\begin{tabular}{|lrrcccccc}
\hline Common name & N & Total no. & Depth & Distance & Substrate & Salinity & DOC & Temperature \\
\hline Macroinvertebrates & & & & & & & \\
Brown shrimp & 267 & 2538 & $1.7 \pm 0.01 \mathrm{~A}$ & $141 \pm 4.1 \mathrm{~A}$ & $2.1 \pm 0.02 \mathrm{~A}$ & $11.0 \pm 0.14 \mathrm{~A}$ & $7.6 \pm 0.02 \mathrm{~A}$ & $26.5 \pm 0.07 \mathrm{~A}$ \\
White shrimp & 140 & 268 & $1.9 \pm 0.04 \mathrm{~A}$ & $230 \pm 15.7 \mathrm{~B}$ & $2.5 \pm 0.06 \mathrm{~B}$ & $13.5 \pm 0.51 \mathrm{~A}$ & $8.6 \pm 0.08 \mathrm{~B}$ & $20.0 \pm 0.30 \mathrm{~B}$ \\
Blue crab & 548 & 3717 & $1.4 \pm 0.01 \mathrm{~B}$ & $152 \pm 3.6 \mathrm{~A}$ & $2.4 \pm 0.02 \mathrm{~B}$ & $9.5 \pm 0.09 \mathrm{~B}$ & $8.5 \pm 0.02 \mathrm{~B}$ & $18.2 \pm 0.10 \mathrm{C}$ \\
Lesser blue crab & 252 & 2074 & $2.3 \pm 0.02 \mathrm{C}$ & $267 \pm 6.2 \mathrm{~B}$ & $3.0 \pm 0.02 \mathrm{C}$ & $19.0 \pm 0.13 \mathrm{C}$ & $8.5 \pm 0.04 \mathrm{~B}$ & $22.7 \pm 0.11 \mathrm{D}$ \\
Roughneck shrimp & 60 & 570 & $2.6 \pm 0.03 \mathrm{D}$ & $430 \pm 12.0 \mathrm{C}$ & $3.0 \pm 0.03 \mathrm{C}$ & $20.9 \pm 0.15 \mathrm{D}$ & $9.5 \pm 0.03 \mathrm{C}$ & $17.8 \pm 0.12 \mathrm{BC}$ \\
Flatfishes & & & & & & & & \\
Bay whiff & 321 & 1583 & $1.4 \pm 0.01 \mathrm{~A}$ & $208 \pm 7.3 \mathrm{~A}$ & $2.1 \pm 0.02 \mathrm{~A}$ & $10.1 \pm 0.14 \mathrm{~A}$ & $8.2 \pm 0.04 \mathrm{~A}$ & $19.7 \pm 0.15 \mathrm{~A}$ \\
Blackcheek tonguefish & 149 & 322 & $1.7 \pm 0.04 \mathrm{~B}$ & $199 \pm 16.7 \mathrm{~A}$ & $2.4 \pm 0.07 \mathrm{~B}$ & $9.9 \pm 0.29 \mathrm{~A}$ & $8.6 \pm 0.06 \mathrm{~B}$ & $20.2 \pm 0.34 \mathrm{AC}$ \\
Offshore tonguefish & 213 & 1512 & $2.3 \pm 0.02 \mathrm{C}$ & $352 \pm 10.2 \mathrm{~B}$ & $2.9 \pm 0.02 \mathrm{C}$ & $14.9 \pm 0.16 \mathrm{~B}$ & $8.9 \pm 0.03 \mathrm{C}$ & $17.9 \pm 0.11 \mathrm{~B}$ \\
Fringed flounder & 206 & 483 & $2.0 \pm 0.03 \mathrm{D}$ & $366 \pm 24.8 \mathrm{~B}$ & $2.6 \pm 0.05 \mathrm{~B}$ & $17.5 \pm 0.27 \mathrm{C}$ & $9.1 \pm 0.06 \mathrm{C}$ & $20.9 \pm 0.25 \mathrm{C}$ \\
Other fishes & & & & & & & & \\
Bay anchovy & 374 & 8833 & $1.8 \pm 0.01 \mathrm{~A}$ & $298 \pm 4.4 \mathrm{~A}$ & $2.2 \pm 0.01 \mathrm{~A}$ & $9.3 \pm 0.07 \mathrm{~A}$ & $8.1 \pm 0.01 \mathrm{~A}$ & $24.7 \pm 0.07 \mathrm{~A}$ \\
Atlantic croaker & 404 & 4327 & $1.6 \pm 0.01 \mathrm{~B}$ & $251 \pm 4.3 \mathrm{~A}$ & $2.4 \pm 0.02 \mathrm{~A}$ & $11.7 \pm 0.09 \mathrm{~B}$ & $9.1 \pm 0.02 \mathrm{~B}$ & $16.4 \pm 0.04 \mathrm{~B}$ \\
Spot & 65 & 452 & $1.2 \pm 0.02 \mathrm{C}$ & $228 \pm 15.0 \mathrm{~B}$ & $2.0 \pm 0.05 \mathrm{~B}$ & $13.5 \pm 0.21 \mathrm{C}$ & $9.1 \pm 0.04 \mathrm{BC}$ & $15.9 \pm 0.20 \mathrm{BC}$ \\
Darter goby & 270 & 1839 & $1.5 \pm 0.01 \mathrm{~B}$ & $264 \pm 13.5 \mathrm{~B}$ & $2.6 \pm 0.03 \mathrm{C}$ & $11.9 \pm 0.15 \mathrm{~B}$ & $9.2 \pm 0.02 \mathrm{C}$ & $15.4 \pm 0.08 \mathrm{C}$ \\
\hline
\end{tabular}
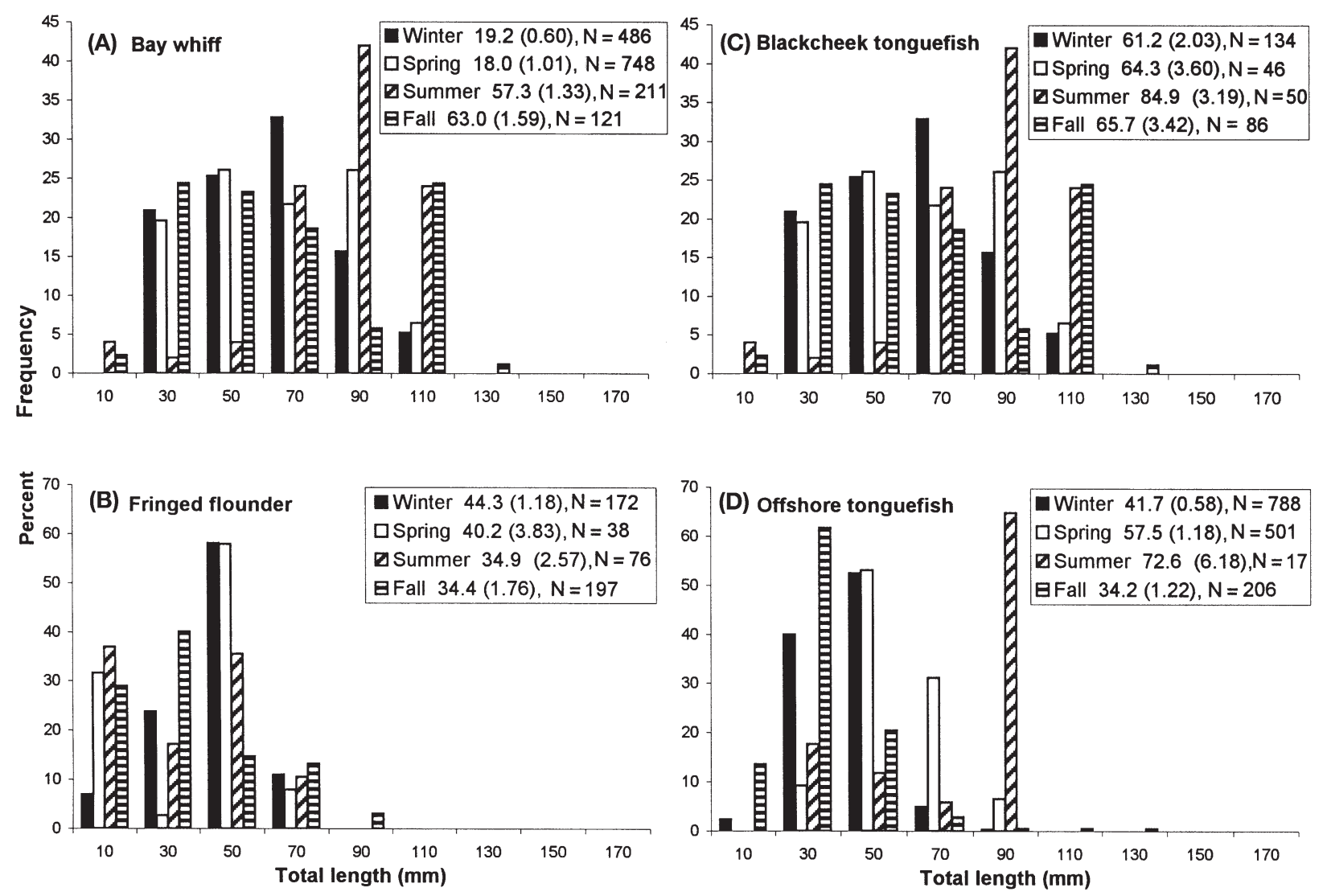

Fig. 3. Seasonal size frequency distributions for 4 common flatfishes in the Barataria Basin: (A) bay whiff, (B) fringed flounder, (C) blackcheek tonguefish and (D) offshore tonguefish. Numbers in legends are mean lengths (SE); $\mathrm{N}=$ total number of individuals 

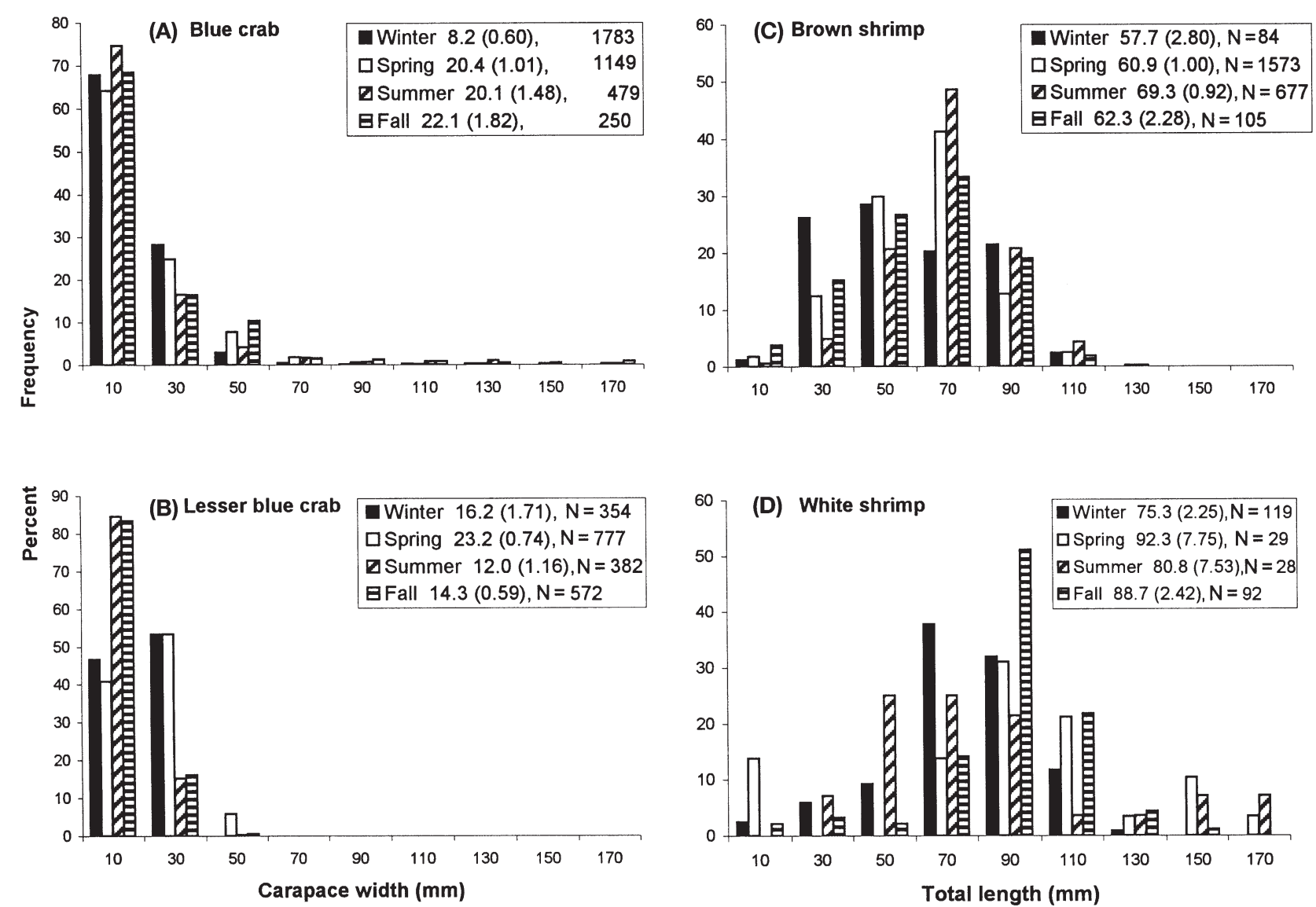

Fig. 4. Seasonal size frequency distributions for 4 common macroinvertebrates in the Barataria Basin: (A) blue crab, (B) lesser blue crab, (C) brown shrimp and (D) white shrimp. Numbers in legends are mean lengths (SE); $\mathrm{N}=$ total number of individuals

exceptions were blue crab, lesser blue crab, bay whiff and darter goby, whose sizes were predominantly $\leq 30 \mathrm{~mm}$. The vast majority of species, including white shrimp, blue crab, roughneck shrimp, Atlantic croaker, spot, blackcheek tonguefish, offshore tonguefish and darter goby, were most abundant as small juveniles in the winter. Brown shrimp and bay whiff recruitment peaked in the spring. Two other species, lesser blue crab and fringed flounder, recruited in the fall, and bay anchovy was the only species whose highest juvenile abundance occurred in the summer.

\section{Among species comparisons}

For individual environmental variables, the 5 common macroinvertebrate species showed significant differences (1-way ANOVAs: $F$-ratio $\geq 31.14, \mathrm{df} \geq 4$ and $1249, p \leq 0.0001$ ) in resource use for all 6 environmental variables (Table 3). Four of 5 macroinvertebrate species differed significantly for depth, salinity and distance. Three species differed significantly for distance, substrate and dissolved oxygen concentration.
The 4 common flatfish species showed significant differences ( $F$-ratio $\geq 12.51, \mathrm{df} \geq 3$ and $878, \mathrm{p} \leq 0.0001$ ) in resource use for all 6 environmental variables (Table 3). All 4 flatfishes differed significantly for depth, bay whiff and blackcheek tonguefish were found significantly closer to shore than fringed flounder and offshore tonguefish, and 3 of 4 species differed for substrate, salinity, dissolved oxygen concentration, and temperature. The other 4 common fish species showed significant differences ( $F$-ratio $\geq 8.13$, df $\geq 3$ and 1104, $p \leq 0.001$ ) in resource use for all 6 environmental variables (Table 3). Bay anchovy and Atlantic croaker were found further from shore than spot and darter goby, and 3 of 4 species differed with regard to depth, substrate, salinity, dissolved oxygen concentration and temperature.

\section{Within species comparisons}

Ontogenetic shifts within species were common. Within the 13 selected species, resource use patterns among size classes differed significantly for at least 
Table 4. Weighted means \pm 1 SE of environmental variables used by size classes of 13 selected (see text) fish and invertebrate species in Barataria Bay, Louisiana. $\mathrm{N}$ is the number of independent samples, and total number is the number of individuals. Posterior testing of overall significant differences $(\mathrm{p} \leq 0.048)$ between size-class means within species is based on LSM comparisons, and significant differences are indicated by different letters (i.e. A, B and C) reading vertically

\begin{tabular}{|c|c|c|c|c|c|c|c|c|}
\hline Common name & $\mathrm{N}$ & Total no. & Depth & Distance & Substrate & Salinity & DOC & Temperature \\
\hline \multicolumn{9}{|l|}{ Brown shrimp } \\
\hline$\leq 15 \mathrm{~mm}$ & 4 & 5 & $1.5 \pm 0.31$ & $32 \pm 8.6$ & $3.0 \pm 0.66$ & $10.4 \pm 4.40$ & $8.0 \pm 0.57$ & $20.4 \pm 3.14$ \\
\hline$\leq 30 \mathrm{~mm}$ & 43 & 137 & $1.2 \pm 0.04 \mathrm{~A}$ & $90 \pm 22.4 \mathrm{~A}$ & $2.3 \pm 0.13$ & $9.8 \pm 0.79$ & $7.7 \pm 0.15$ & $21.3 \pm 0.88 \mathrm{~A}$ \\
\hline$\leq 100 \mathrm{~mm}$ & 184 & 2331 & $1.7 \pm 0.03 \mathrm{~B}$ & $139 \pm 14.4 \mathrm{~B}$ & $1.9 \pm 0.08$ & $9.8 \pm 0.50$ & $7.5 \pm 0.08$ & $26.5 \pm 0.24 \mathrm{~B}$ \\
\hline$>100 \mathrm{~mm}$ & 36 & 65 & $1.9 \pm 0.06 \mathrm{~B}$ & $162 \pm 30.6 \mathrm{AB}$ & $2.1 \pm 0.16$ & $13.5 \pm 0.99$ & $7.7 \pm 0.15$ & $27.1 \pm 0.44 \mathrm{~B}$ \\
\hline \multicolumn{9}{|l|}{ White shrimp } \\
\hline$\leq 15 \mathrm{~mm}$ & 4 & 5 & $1.2 \pm 0.10$ & $82 \pm 23.6$ & $2.5 \pm 0.14$ & $6.2 \pm 0.90$ & $8.2 \pm 0.62$ & $18.1 \pm 1.67$ \\
\hline$\leq 30 \mathrm{~mm}$ & 10 & 12 & $1.4 \pm 0.11$ & $69 \pm 17.4$ & $2.1 \pm 0.16$ & $8.1 \pm 1.54$ & $8.2 \pm 0.49$ & $19.8 \pm 1.66$ \\
\hline$\leq 100 \mathrm{~mm}$ & 85 & 195 & $1.8 \pm 0.06 \mathrm{~A}$ & $212 \pm 27.5$ & $2.3 \pm 0.10$ & $11.1 \pm 0.88 \mathrm{~A}$ & $8.6 \pm 0.14$ & $19.0 \pm 0.45 \mathrm{~A}$ \\
\hline$>100 \mathrm{~mm}$ & 41 & 56 & $2.1 \pm 0.12 \mathrm{~B}$ & $249 \pm 38.2$ & $2.7 \pm 0.20$ & $16.3 \pm 1.22 \mathrm{~B}$ & $8.5 \pm 0.23$ & $22.4 \pm 0.76 \mathrm{~B}$ \\
\hline \multicolumn{9}{|l|}{ Blue crab } \\
\hline$\leq 15 \mathrm{~mm}$ & 171 & 2022 & $1.3 \pm 0.03 \mathrm{~A}$ & $126 \pm 13.5 \mathrm{~A}$ & $2.3 \pm 0.07$ & $8.7 \pm 0.39$ & $8.4 \pm 0.10 \mathrm{~A}$ & $17.3 \pm 0.42 \mathrm{~A}$ \\
\hline$\leq 30 \mathrm{~mm}$ & 183 & 1190 & $1.4 \pm 0.03 \mathrm{~B}$ & $160 \pm 16.6 \mathrm{~B}$ & $2.5 \pm 0.09$ & $8.6 \pm 0.36$ & $8.9 \pm 0.08 \mathrm{~B}$ & $16.1 \pm 0.40 \mathrm{~B}$ \\
\hline$\leq 100 \mathrm{~mm}$ & 172 & 473 & $1.7 \pm 0.04 \mathrm{C}$ & $168 \pm 16.3 \mathrm{AB}$ & $2.6 \pm 0.11$ & $10.6 \pm 0.52$ & $8.4 \pm 0.10 \mathrm{~A}$ & $20.0 \pm 0.48 \mathrm{C}$ \\
\hline$>100 \mathrm{~mm}$ & 22 & 32 & $1.7 \pm 0.07 \mathrm{BC}$ & $262 \pm 72.1 \mathrm{AB}$ & $2.2 \pm 0.25$ & $6.9 \pm 1.20$ & $7.5 \pm 0.24 \mathrm{~A}$ & $24.8 \pm 1.41 \mathrm{C}$ \\
\hline \multicolumn{9}{|l|}{ Lesser blue crab } \\
\hline$\leq 15 \mathrm{~mm}$ & 106 & 1008 & $1.9 \pm 0.09 \mathrm{~A}$ & $223 \pm 25.6 \mathrm{~A}$ & $2.8 \pm 0.11 \mathrm{~A}$ & $18.4 \pm 0.66$ & $8.9 \pm 0.14 \mathrm{~A}$ & $23.4 \pm 0.48 \mathrm{~A}$ \\
\hline$\leq 30 \mathrm{~mm}$ & 111 & 853 & $2.4 \pm 0.09 B$ & $308 \pm 27.9 B$ & $3.1 \pm 0.11 \mathrm{~B}$ & $17.9 \pm 0.56$ & $8.6 \pm 0.15 \mathrm{~A}$ & $21.0 \pm 0.50 \mathrm{~B}$ \\
\hline$\leq 100 \mathrm{~mm}$ & 35 & 224 & $3.0 \pm 0.10 \mathrm{C}$ & $251 \pm 46.1 \mathrm{~B}$ & $3.3 \pm 0.09 \mathrm{~B}$ & $19.7 \pm 0.54$ & $6.5 \pm 0.29 \mathrm{~B}$ & $23.8 \pm 0.63 \mathrm{~A}$ \\
\hline \multicolumn{9}{|c|}{ Roughneck shrimp } \\
\hline$\leq 15 \mathrm{~mm}$ & 5 & 25 & $3.1 \pm 0.09$ & $414 \pm 83.1$ & $2.9 \pm 0.21$ & $27.0 \pm 0.75$ & $10.5 \pm 0.21$ & $23.7 \pm 0.73$ \\
\hline$\leq 30 \mathrm{~mm}$ & 28 & 173 & $2.5 \pm 0.11$ & $368 \pm 45.2$ & $3.0 \pm 0.11$ & $21.6 \pm 1.06$ & $9.7 \pm 0.16$ & $18.8 \pm 0.68 \mathrm{~A}$ \\
\hline$\leq 100 \mathrm{~mm}$ & 27 & 372 & $2.6 \pm 0.12$ & $460 \pm 59.8$ & $3.1 \pm 0.12$ & $20.0 \pm 0.44$ & $9.4 \pm 0.15$ & $16.9 \pm 0.33 \mathrm{~B}$ \\
\hline \multicolumn{9}{|l|}{ Bay anchovy } \\
\hline$\leq 15 \mathrm{~mm}$ & 7 & 10 & $1.5 \pm 0.24$ & $127 \pm 116.4$ & $2.2 \pm 0.35$ & $12.0 \pm 0.52$ & $8.0 \pm 0.44$ & $28.2 \pm 1.21$ \\
\hline$\leq 30 \mathrm{~mm}$ & 149 & 3377 & $1.5 \pm 0.04 \mathrm{~A}$ & $185 \pm 23.8 \mathrm{~A}$ & $1.9 \pm 0.07 \mathrm{~A}$ & $7.8 \pm 0.37$ & $8.0 \pm 0.11$ & $25.7 \pm 0.55 \mathrm{~A}$ \\
\hline$\leq 100 \mathrm{~mm}$ & 218 & 5446 & $1.9 \pm 0.04 \mathrm{~B}$ & $315 \pm 29.6 B$ & $2.2 \pm 0.07 \mathrm{~B}$ & $9.6 \pm 0.45$ & $8.2 \pm 0.09$ & $22.4 \pm 0.47 \mathrm{~B}$ \\
\hline \multicolumn{9}{|l|}{ Atlantic croaker } \\
\hline$\leq 15 \mathrm{~mm}$ & 67 & 186 & $1.6 \pm 0.08$ & $219 \pm 40.7 \mathrm{AB}$ & $2.6 \pm 0.11 \mathrm{~A}$ & $11.6 \pm 0.83$ & $9.1 \pm 0.12$ & $16.5 \pm 0.20 \mathrm{AB}$ \\
\hline$\leq 30 \mathrm{~mm}$ & 153 & 1612 & $1.6 \pm 0.05$ & $202 \pm 21.3 \mathrm{~A}$ & $2.4 \pm 0.08 \mathrm{~A}$ & $10.5 \pm 0.51$ & $9.0 \pm 0.09$ & $16.7 \pm 0.14 \mathrm{~A}$ \\
\hline$\leq 100 \mathrm{~mm}$ & 159 & 2491 & $1.5 \pm 0.04$ & $259 \pm 23.0 \mathrm{~B}$ & $2.1 \pm 0.09 B$ & $10.7 \pm 0.41$ & $9.0 \pm 0.09$ & $15.7 \pm 0.27 \mathrm{~B}$ \\
\hline$>100 \mathrm{~mm}$ & 25 & 38 & $1.8 \pm 0.09$ & $158 \pm 33.8 \mathrm{AB}$ & $1.8 \pm 0.24 \mathrm{AB}$ & $39.8 \pm 1.23$ & $7.6 \pm 0.24$ & $24.9 \pm 0.94 \mathrm{C}$ \\
\hline \multicolumn{9}{|l|}{ Spot } \\
\hline$\leq 15 \mathrm{~mm}$ & 1 & 1 & $1.0-$ & $10-$ & $4.1-$ & $19.6-$ & $8.3-$ & $17.0-$ \\
\hline$\leq 30 \mathrm{~mm}$ & 4 & 4 & $1.3 \pm 0.18$ & $44 \pm 12.1$ & $2.8 \pm 0.80$ & $13.3 \pm 1.15$ & $8.8 \pm 0.51$ & $16.8 \pm 3.15$ \\
\hline$\leq 100 \mathrm{~mm}$ & 37 & 416 & $1.1 \pm 0.04 \mathrm{~A}$ & $241 \pm 55.0$ & $1.8 \pm 0.18$ & $13.0 \pm 0.70$ & $9.3 \pm 0.12 \mathrm{~A}$ & $14.9 \pm 0.45 \mathrm{~A}$ \\
\hline$>100 \mathrm{~mm}$ & 23 & 31 & $1.7 \pm 0.10 \mathrm{~B}$ & $258 \pm 58.4$ & $2.6 \pm 0.23$ & $12.4 \pm 0.78$ & $7.7 \pm 0.27 \mathrm{~B}$ & $25.6 \pm 1.32 \mathrm{~B}$ \\
\hline \multicolumn{9}{|l|}{ Bay whiff } \\
\hline$\leq 15 \mathrm{~mm}$ & 87 & 580 & $1.4 \pm 0.05 \mathrm{~A}$ & $170 \pm 23.4$ & $2.2 \pm 0.09 \mathrm{~A}$ & $11.0 \pm 0.57 \mathrm{~A}$ & $8.3 \pm 0.14 \mathrm{~A}$ & $16.7 \pm 0.34 \mathrm{~A}$ \\
\hline$\leq 30 \mathrm{~mm}$ & 93 & 570 & $1.3 \pm 0.03 \mathrm{~B}$ & $219 \pm 32.5$ & $2.0 \pm 0.08 \mathrm{~B}$ & $8.9 \pm 0.43 \mathrm{~B}$ & $8.3 \pm 0.14 \mathrm{~A}$ & $17.4 \pm 0.51 \mathrm{~A}$ \\
\hline$\leq 100 \mathrm{~mm}$ & 141 & 433 & $1.6 \pm 0.04 \mathrm{C}$ & $196 \pm 23.9$ & $1.9 \pm 0.07 \mathrm{~B}$ & $8.8 \pm 0.47 \mathrm{~B}$ & $7.8 \pm 0.12 \mathrm{~B}$ & $25.8 \pm 0.46 \mathrm{~B}$ \\
\hline \multicolumn{9}{|c|}{ Blackcheek tonguefish } \\
\hline$\leq 15 \mathrm{~mm}$ & 1 & 1 & $1.7-$ & $75-$ & $2.5-$ & $8.0-$ & $9.2-$ & $17.9-$ \\
\hline$\leq 30 \mathrm{~mm}$ & 19 & 26 & $1.4 \pm 0.11 \mathrm{~A}$ & $151 \pm 35.1 \mathrm{~A}$ & $2.1 \pm 0.20$ & $8.0 \pm 0.93 \mathrm{~A}$ & $8.9 \pm 0.27$ & $17.4 \pm 0.89 \mathrm{~A}$ \\
\hline$\leq 100 \mathrm{~mm}$ & 103 & 253 & $1.6 \pm 0.05 \mathrm{~A}$ & $162 \pm 28.5 \mathrm{~A}$ & $2.4 \pm 0.12$ & $8.6 \pm 0.44 \mathrm{~A}$ & $8.6 \pm 0.10$ & $19.2 \pm 0.55 \mathrm{~A}$ \\
\hline$>100 \mathrm{~mm}$ & 26 & 42 & $2.2 \pm 0.16 \mathrm{~B}$ & $340 \pm 63.4 \mathrm{~B}$ & $2.8 \pm 0.26$ & $15.5 \pm 0.79 \mathrm{~B}$ & $8.5 \pm 0.30$ & $24.6 \pm 1.06 \mathrm{~B}$ \\
\hline \multicolumn{9}{|c|}{ Offshore tonguefish } \\
\hline$\leq 15 \mathrm{~mm}$ & 11 & 19 & $2.5 \pm 0.36$ & $594 \pm 229.6$ & $2.1 \pm 0.13$ & $19.9 \pm 2.55$ & $9.6 \pm 0.34$ & $19.0 \pm 1.07$ \\
\hline$\leq 30 \mathrm{~mm}$ & 60 & 184 & $1.9 \pm 0.09$ & $338 \pm 82.3$ & $2.4 \pm 0.11$ & $11.3 \pm 0.84$ & $9.4 \pm 0.10$ & $15.6 \pm 0.52 \mathrm{~A}$ \\
\hline$\leq 100 \mathrm{~mm}$ & 140 & 1307 & $2.1 \pm 0.06$ & $327 \pm 31.4$ & $2.8 \pm 0.08$ & $13.9 \pm 0.53$ & $8.9 \pm 0.10$ & $17.4 \pm 0.34 \mathrm{~B}$ \\
\hline$>100 \mathrm{~mm}$ & 2 & 2 & $2.4 \pm 1.13$ & $830 \pm 779.5$ & $2.3 \pm 1.20$ & $15.7 \pm 3.85$ & $8.4 \pm 1.85$ & $26.5 \pm 2.50$ \\
\hline \multicolumn{9}{|l|}{ Fringed flounder } \\
\hline$\leq 15 \mathrm{~mm}$ & 33 & 59 & $2.0 \pm 0.11$ & $179 \pm 43.9 \mathrm{~A}$ & $2.5 \pm 0.18 \mathrm{AB}$ & $15.4 \pm 1.02$ & $9.0 \pm 0.19$ & $23.6 \pm 1.02 \mathrm{~A}$ \\
\hline$\leq 30 \mathrm{~mm}$ & 60 & 130 & $1.9 \pm 0.07$ & $421 \pm 96.5 B$ & $2.2 \pm 0.10 \mathrm{~A}$ & $18.4 \pm 0.88$ & $9.4 \pm 0.14$ & $21.1 \pm 0.60 \mathrm{~A}$ \\
\hline$\leq 100 \mathrm{~mm}$ & 113 & 294 & $1.9 \pm 0.07$ & $416 \pm 58.0 B$ & $2.7 \pm 0.10 \mathrm{~B}$ & $16.9 \pm 0.58$ & $9.0 \pm 0.12$ & $19.5 \pm 0.53 \mathrm{~B}$ \\
\hline
\end{tabular}


Table 4 (continued)

\begin{tabular}{|lrrrrrrrr}
\hline Common name & N & Total no. & Depth & Distance & Substrate & Salinity & DOC & Temperature \\
\hline $\begin{array}{l}\text { Darter goby } \\
\leq 15 \mathrm{~mm}\end{array}$ & 9 & 13 & $1.7 \pm 0.16$ & $294 \pm 172.4$ & $2.4 \pm 0.26$ & $12.0 \pm 3.00$ & $8.9 \pm 0.60$ & $16.5 \pm 0.98$ \\
$\leq 30 \mathrm{~mm}$ & 121 & 868 & $1.5 \pm 0.04$ & $270 \pm 56.4$ & $2.5 \pm 0.10$ & $11.5 \pm 0.54$ & $9.4 \pm 0.10$ & $15.0 \pm 0.31$ \\
$\leq 100 \mathrm{~mm}$ & 140 & 958 & $1.5 \pm 0.04$ & $286 \pm 50.0$ & $2.5 \pm 0.11$ & $11.0 \pm 0.53$ & $9.2 \pm 0.08$ & $15.1 \pm 0.28$ \\
\hline
\end{tabular}

1 environmental variable for all except the darter goby, the smallest species (Table 4). For bay anchovy, the 2 intermediate size classes (SC2 and SC3) differed significantly (1-way ANOVAs: F-ratio $\geq 6.86$, df $\geq 1$ and $364, \mathrm{p} \leq$ 0.0092) in depth, distance from shore, substrate and temperature (Table 4). Bay anchovy showed a clear pattern of changing resource use with increasing size. Larger bay anchovy used deeper water further from shore, coarser substrates and cooler temperatures. They were collected from every stratum in salinities ranging from 0.9 to 29.2 psu. Recruitment of juveniles $\leq 20 \mathrm{~mm}$ took place year around, but peaks in overall abundance and recruitment occurred in summer (Fig. 2A). Atlantic croaker showed significant differences (1-way ANOVAs: F-ratio $\geq 2.97$, df $\geq 3$ and $398, \mathrm{p} \leq 0.0317$ ) in patterns of use for distance from shore, substrate, and temperature (Table 4). Atlantic croaker were sampled in every stratum from salinities of 0.9 psu in the upper study area to 29.1 psu in Stratum VI. Recruitment of juveniles $\leq 20 \mathrm{~mm}$ began in the fall and peaked in the winter (Fig. 2B). For spot, the 2 largest size classes showed significant differences (1-way ANOVAs: F-ratio $\geq 11.87, \mathrm{df}=1$ and 58, $\mathrm{p} \leq$ 0.0011 ) in depth, dissolved oxygen and temperature (Table 4). The largest size class used deeper water, lower dissolved oxygen concentrations and higher temperatures, and was present in the basin mainly during the summer (Fig. 2C). Spot were collected all along the salinity gradient from 6.0 to $22.0 \mathrm{psu}$, but were most concentrated in Strata III to V. Peak abundance of spot corresponded to that of Atlantic croaker in winter; however, the size structures of the 2 populations were different (Fig. 2B,C). Darter goby showed no significantly different patterns of resource use (1-way ANOVAs: F-ratio $\leq 2.21$, df $\geq 1$ and 257, p $\geq$ 0.1380 ) (Table 4). They were collected from every stratum with salinity ranging from 0.9 to $29.1 \mathrm{psu}$, but were only found in Strata IV and V in the summer. Their abundance was highest in the winter, but changes in the size structure for this small species were poorly documented by the size classes we used (Fig. 2D).

Differences in patterns of resource use occurred for all variables within the 4 common flatfishes, and temperature differences were universal; however, dissolved oxygen concentrations differed in only 1 spe- cies. The 3 smallest size classes of bay whiff showed significantly different (1-way ANOVAs: F-ratio $\geq 4.83$, df $\geq 2$ and 316, $\mathrm{p} \leq 0.0085$ ) resource use patterns for depth, substrate, salinity, dissolved oxygen and temperature (Table 4). Recruitment was greatest in the winter and spring (Fig. 3A), so growing juveniles experienced increasing temperature and decreasing dissolved oxygen concentrations as they progressed through size classes. Bay whiff abundance was highest in the spring and samples were collected in every stratum in every season from salinities of 0.9 to $28.1 \mathrm{psu}$. The 3 smaller size classes of fringed flounder differed significantly (1-way ANOVAs: F-ratio $\geq 3.35$, df $\geq 2$ and $199, p \leq 0.0369$ ) in patterns of resource use for distance from shore, substrate and temperature (Table 4). Fringed flounder were collected in all strata from salinities as low as $2.5 \mathrm{psu}$ to as high as $29.2 \mathrm{psu}$, but were absent from spring samples of the upper 3 strata. Recruitment appeared to take place in summer and fall for juveniles $\leq 20 \mathrm{~mm}$, while overall abundance peaked in fall and winter (Fig. 3B). Resource use patterns of blackcheek tonguefish differed significantly (1-way ANOVAs: $F$-ratio $\geq 4.58, \mathrm{df}=2$ and $145, \mathrm{p} \leq 0.0118$ ) for depth, distance from shore, salinity and temperature (Table 4). Blackcheek tonguefish were found primarily in Strata II to IV, although they occurred in all strata in salinities ranging from $2.3 \mathrm{psu}$ in Stratum I to 28.3 in Stratum VI. Recruitment began in the fall, and overall, abundance peaked in the winter (Fig. 3C). The 2 intermediate size classes (SC2 and SC3) of offshore tonguefish differed significantly (1-way ANOVAs: F-ratio = 6.64, df $=1$ and 198, $\mathrm{p} \leq 0.0107$ ) only for temperature (Table 4) and the larger individuals (SC3) used warmer water. Although sampled in all strata from salinities of 3.0 to $29.2 \mathrm{psu}$, offshore tonguefish were most abundant in Strata V and VI. Like blackcheek tonguefish, recruitment began in the fall, and abundance peaked in winter (Fig. 3D). In contrast, offshore tonguefish were more localized and more abundant than blackcheek tonguefish.

Within the 2 congeneric blue crabs, differences in patterns of resource use occurred for all variables except salinity, but substrate differences were only detected in the lesser blue crab. The pattern of resource use for blue crab size classes differed significantly 
Table 5. Proportional overlaps in distributions of selected fish and invertebrate species (see text) based on the temporal (4 seasons) and spatial (6 strata) occurrence of 4 size classes. Overlaps above the diagonal are based on seasonal, spatial and size class distributions. Overlaps below the diagonal are only for seasonal and spatial distributions

\begin{tabular}{|c|c|c|c|c|c|c|c|c|c|c|c|c|c|}
\hline Species & $\begin{array}{l}\text { Brown } \\
\text { shrimp }\end{array}$ & $\begin{array}{l}\text { White } \\
\text { shrimp }\end{array}$ & $\begin{array}{l}\text { Blue } \\
\text { crab }\end{array}$ & $\begin{array}{l}\text { Lesser } \\
\text { blue } \\
\text { crab }\end{array}$ & $\begin{array}{c}\text { Rough- } \\
\text { neck } \\
\text { shrimp }\end{array}$ & $\begin{array}{c}\text { Bay } \\
\text { anchovy }\end{array}$ & $\begin{array}{l}\text { Atlantic } \\
\text { croaker }\end{array}$ & Spot & $\begin{array}{l}\text { Bay } \\
\text { whiff }\end{array}$ & $\begin{array}{l}\text { Black- } \\
\text { cheek } \\
\text { tonguefish }\end{array}$ & $\begin{array}{l}\text { Offshore } \\
\text { tongue- } \\
\text { fish }\end{array}$ & $\begin{array}{l}\text { Fringed } \\
\text { flounder }\end{array}$ & $\begin{array}{c}\text { Darter } \\
\text { goby }\end{array}$ \\
\hline Brown shrimp & - & 0.19 & 0.16 & 0.06 & 0.02 & 0.31 & 0.23 & 0.20 & 0.24 & 0.27 & 0.10 & 0.18 & 0.23 \\
\hline White shrimp & 0.21 & - & 0.16 & 0.07 & 0.14 & 0.41 & 0.34 & 0.10 & 0.23 & 0.51 & 0.44 & 0.36 & 0.30 \\
\hline Blue crab & 0.40 & 0.55 & - & 0.23 & 0.05 & 0.25 & 0.33 & 0.07 & 0.59 & 0.17 & 0.20 & 0.18 & 0.34 \\
\hline Lesser blue crab & 0.32 & 0.41 & 0.26 & - & 0.04 & 0.09 & 0.19 & 0.02 & 0.18 & 0.04 & 0.16 & 0.23 & 0.14 \\
\hline Roughneck shrimp & 0.03 & 0.24 & 0.06 & 0.04 & - & 0.13 & 0.17 & 0.01 & 0.03 & 0.03 & 0.43 & 0.2 & 0.04 \\
\hline Bay anchovy & 0.39 & 0.58 & 0.50 & 0.38 & 0.15 & - & 0.44 & 0.13 & 0.26 & 0.36 & 0.35 & 0.4 & 0.33 \\
\hline Atlantic croaker & 0.27 & 0.57 & 0.71 & 0.31 & 0.17 & 0.52 & - & 0.38 & 0.29 & 0.40 & 0.29 & 0.33 & 0.58 \\
\hline Spot & 0.26 & 0.14 & 0.47 & 0.25 & 0.02 & 0.18 & 0.48 & - & 0.06 & 0.21 & 0.19 & 0.17 & 0.31 \\
\hline Bay whiff & 0.43 & 0.43 & 0.72 & 0.26 & 0.06 & 0.45 & 0.56 & 0.61 & - & 0.21 & 0.19 & 0.17 & 0.33 \\
\hline $\begin{array}{l}\text { Blackcheek } \\
\text { tonguefish }\end{array}$ & 0.33 & 0.59 & 0.61 & 0.26 & 0.06 & 0.55 & 0.52 & 0.25 & 0.47 & - & 0.35 & 0.22 & 0.38 \\
\hline $\begin{array}{l}\text { Offshore } \\
\text { tonguefish }\end{array}$ & 0.11 & 0.56 & 0.45 & 0.48 & 0.45 & 0.40 & 0.56 & 0.28 & 0.38 & 0.40 & - & 0.48 & 0.45 \\
\hline Fringed flounder & 0.21 & 0.51 & 0.30 & 0.53 & 0.23 & 0.45 & 0.42 & 0.26 & 0.32 & 0.33 & 0.51 & - & 0.33 \\
\hline Darter goby & 0.26 & 0.40 & 0.59 & 0.31 & 0.04 & 0.37 & 0.64 & 0.61 & 0.48 & 0.47 & 0.46 & 0.37 & - \\
\hline
\end{tabular}

(1-way ANOVAs: F-value $\geq 2.64, \mathrm{df} \geq 3$ and $542, \mathrm{p} \leq$ 0.0488) in depth, distance from the shore, dissolved oxygen concentration and temperature (Table 4). Since recruitment began in fall and abundance peaked in winter (Fig. 4A), blue crab experienced colder, more highly oxygenated water as they grew from SC1 to $\mathrm{SC} 2$. Blue crab were collected in all strata in salinities ranging from 0.9 to $29.2 \mathrm{psu}$, but were most abundant in Strata II to IV. For lesser blue crab, only individuals $\leq 60 \mathrm{~mm}$ were collected. At least 1 size class differed significantly (1-way ANOVAs: F-ratio $\geq 3.86$, $\mathrm{df} \geq 2$ and $243, p \leq 0.0223$ ) for every environmental variable measured except salinity (Table 4). Lesser blue crab were most abundant in spring and fall (Fig. 4b) and the majority were collected in Strata V and VI. They were

Table 6. Rotated factor loadings for environmental variables in Barataria Basin, Louisiana. Magnitude and signs of loadings indicate strength and direction of each variable's influence on a factor. Underlined loadings were used to characterize factors

\begin{tabular}{|lccc|}
\hline & \multicolumn{3}{c|}{ Rotated factor pattern } \\
\cline { 2 - 4 } Environmental variable & 1: Seasonality & $\begin{array}{c}\text { 2: Depth- } \\
\text { Distance }\end{array}$ & $\begin{array}{c}\text { 3: Substrate- } \\
\text { Salinity }\end{array}$ \\
\hline Dissolved oxygen & $-\underline{0.85}$ & 0.14 & 0.09 \\
Temperature & $\underline{\underline{0.87}}$ & 0.10 & -0.07 \\
Depth & 0.20 & $\underline{0.69}$ & 0.33 \\
Distance from shore & -0.20 & $\underline{0.84}$ & -0.08 \\
Substrate & -0.16 & -0.10 & $\underline{0.84}$ \\
Salinity & -0.01 & 0.35 & $\underline{0.68}$ \\
Eigenvalue & 1.8 & 1.5 & 1.0 \\
Variance explained (\%) & 30 & 24 & 16 \\
Cumulative variance (\%) & 30 & 54 & 70 \\
\hline
\end{tabular}

generally found in higher-salinity water than the blue

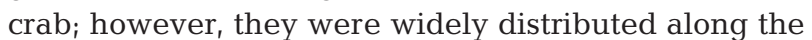
salinity gradient from 3.5 to 29.2 psu, and a small portion $(1.4 \%)$ of the catch occurred in the uppermost stratum.

Differences in patterns of resource use occurred for 4 variables within the 3 common shrimp species. Temperature differences were universal, but differences in substrate and dissolved oxygen concentrations were not detected. For brown shrimp, the 3 larger size classes (SC2 to SC4) differed significantly (1-way ANOVAs: $F$-ratio $\geq 3.92$, df $\geq 2$ and 258, p $\leq 0.0211$ ) in depth, distance from shore and temperature (Table 4). Brown shrimp were collected all along the salinity gradient from $1.8 \mathrm{psu}$ in Stratum I to $29.1 \mathrm{psu}$ in Stratum VI, where they were rare. The majority of individuals were large (40 to $80 \mathrm{~mm}$ ), found in Strata I to V, and most abundant in the spring and summer (Fig. 4C). For white shrimp, the 2 largest size classes (SC3 and SC4) differed significantly (1-way ANOVAs: $F$-ratio $\geq 4.92, \mathrm{df}=2$ and $133, \mathrm{p} \leq 0.0035$ ) in their use of water depth, salinity and temperature (Table 4). White shrimp were collected in all strata with salinities ranging from 2.0 to $29.2 \mathrm{psu}$. Most of the white shrimp caught were large individuals found near the mouth of the estuary, Strata $\mathrm{V}$ and VI, in fall and winter (Fig. 4D). Compared to brown shrimp, white shrimp were much more localized as well as less abundant. All roughneck shrimp in the samples were $<100 \mathrm{~mm}$, and the only significant dif- 
ference in resource use among size classes (1-way ANOVAs: $F$-ratio $=7.43, \mathrm{df}=1$ and $53, \mathrm{p} \leq 0.0087$ ) was a clear ontogenetic shift toward decreasing temperature with increasing size (Table 4). Recruitment of individuals $<30 \mathrm{~mm}$ began in the fall, and abundance peaked in winter. Although roughneck shrimp were found in 5 of the 6 strata in salinities from 5.9 to $28.3 \mathrm{psu}$, they generally occurred at the higher end of the salinity gradient (i.e. $>95 \%$ collected in Stratum VI). greater than or equal to 1 , explained $70 \%$ of the variance, and each of the 6 environmental variables loaded heavily $(\geq 0.68)$ on only 1 axis (Table 6 ). Temperature and dissolved oxygen loaded heavily on Factor 1 but with opposite signs that can be characterized as a negative seasonal relationship. Distance from shore and depth loaded heavily on Factor 2 and were positively related reflecting a local spatial gradient of depth increasing with distance from shore. Factor 3 loaded

\section{Spatial and temporal overlap}

Pairwise overlaps in seasonal and spatial (i.e. strata) distributions of the size-structured assemblage were fairly low (Table 5) and ranged from 0.01 to 0.59 with a mean of 0.24 (median $=0.22$, mode $=0.23)$. Only 22 of 78 overlaps $(28 \%)$ were $\geq 0.33$. The 5 highest pairwise overlaps were blue crab and bay whiff (0.59), Atlantic croaker and darter goby (0.58), white shrimp and blackcheek tonguefish (0.51), offshore tonguefish and fringed flounder (0.48), and offshore tonguefish and darter goby (0.45). For closely related species, the proportional overlap was highest between Atlantic croaker and spot (0.38), slightly lower between blackcheek and offshore tonguefishes (0.35), even lower between blue and lesser blue crabs (0.23) and lowest between brown and white shrimps (0.19).

When size structure was omitted, the seasonal and spatial patterns of overlap in distribution were somewhat higher (Table 5), ranging from 0.02 to 0.72 with a mean of 0.37 (median $=0.40$, mode $=0.26)$. Forty-six of 78 pairwise overlaps $(59 \%)$ were $\geq 0.33$. The top 6 were between bay whiff and blue crab (0.72), Atlantic croaker and blue crab (0.71), Atlantic croaker and darter goby (0.64), spot and bay whiff (0.61), spot and darter goby (0.61) and blackcheek tonguefish and blue crab (0.61). Between closely related species overlaps were relatively low: Atlantic croaker and spot (0.48), blackcheek and offshore tonguefishes (0.40), blue and lesser blue crabs (0.26) and brown and white shrimps (0.21).

\section{3-dimensional environmental space}

In the factor analysis of environmental variables, the first 3 factors had eigenvalues
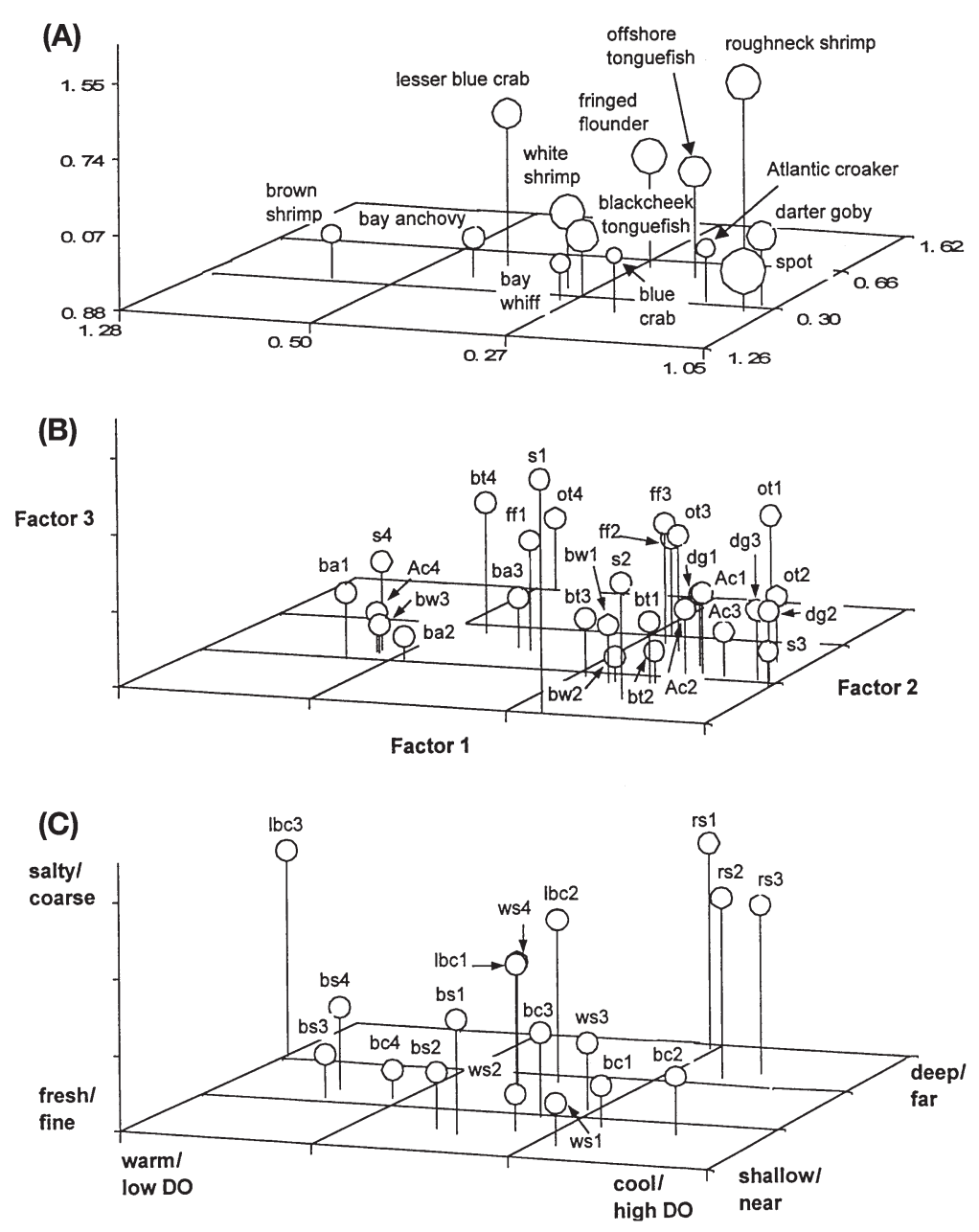

Fig. 5. (A) Resource use pattern of 13 selected fish and macroinvertebrate species in Barataria Basin, Louisiana. The locations of centroids of each species are plotted in 3-dimensional factor space with balloon radii representing $1 \mathrm{SE}$ about the mean. Each factor represents the weighted linear combination of the original set of 6 environmental variables (see text). (B) Resource use patterns of size classes of selected fishes in 3-dimensional factor space. Balloons indicate locations of centroids, but their size is not scaled as a measure of variance. Species codes are: ba = bay anchovy, $\mathrm{Ac}=$ Atlantic croaker, $\mathrm{s}=$ spot, $\mathrm{bw}=$ bay whiff, bt $=$ blackcheek tonguefish, ot $=$ offshore tonguefish, $\mathrm{ff}=$ fringed flounder and $\mathrm{dg}=$ darter goby. (C) Resource use patterns of size classes of selected macroinvertebrates in 3-dimensional factor space. Balloons indicate locations of centroids but are not scaled as a measure of variance. Species codes are: bs = brown shrimp, ws = white shrimp, bc = blue crab, lbc = lesser blue crab and rs = roughneck shrimp 
heavily for substrate and salinity, which were both positively related in a large scale spatial gradient of increasing particle size closer to the Gulf of Mexico, where wave energy reworks the sediment and salinities are higher.

The 13 selected species and size classes plotted in a 3-dimensional environmental space reflect seasonal and spatial resource use differences in the Barataria Basin assemblage (Fig. 5A). Species which were most abundant in winter, e.g. darter goby, Atlantic croaker and spot, were associated in cooler water with high dissolved oxygen. Species like bay anchovy and brown shrimp whose abundance peaked in spring or summer were associated in relatively warmer, less oxygenated water. Lesser blue crab and roughneck shrimp were associated over coarser substrates in higher-salinity water.

There were several ontogenetic shifts apparent when size classes of selected fish species were plotted in 3-dimensional environmental space (Fig. 5B). Some of the differences, especially along the seasonal axis (Factor 1), may have resulted from seasonal changes in environmental conditions (Table 1) and the brief duration of size classes. Other ontogenetic shifts were more notable. Small Atlantic croaker showed only minor differences in resource use until the largest size class (SC4) shifted to much warmer water with a lower dissolved oxygen concentration. Similarly, the largest size classes of spot and bay whiff also shifted to much warmer, less oxygenated water. Blackcheek tonguefish made a large shift between SC3 and SC4 to warmer, less oxygenated water that was deeper and further from shore, higher in salinity and over coarser substrates. The 2 larger size classes of fringed flounder were found in cooler water with higher dissolved oxygen concentration compared to the smallest size class (SC1).

Ontogenetic shifts in resource use were also apparent when size classes of selected macroinvertebrates were plotted in environmental space (Fig. 5C). The 3 larger size classes (SC2 to SC4) of white shrimp shifted to progressively deeper water further from shore, but they also shifted to higher salinities over coarser substrates. Blue crab shifted only along the seasonal axis and in both directions, probably the result of size classes experiencing different seasons instead of an ontogenetic shift. The largest individuals (SC3) of lesser blue crab were found in water that was much warmer and less oxygenated than that used by the other 2 size classes. Size classes of roughneck shrimp were tightly clustered in environmental space away from other species, but there was a slight shift to shallower, nearshore water and then to cooler water with higher dissolved oxygen concentrations as they grew.

\section{DISCUSSION}

If habitat is viewed as the range of environmental conditions used by a species or life history stage (Baltz 1990), then the quantity and quality of suitable habitat should vary seasonally and annually with changing environmental conditions and with continuing anthropogenic and natural changes. The concepts of habitat suitability and essential fish habitat include a notion of quality (Baltz 1990, Minello 1999). We are not just asking where a species lives, but where it lives well. In other words, where are the resource needs (sensu Hurlbert 1981, 'realized niche': resources used; energy, materials and sites) of species and life history stages best met? This question can be addressed by determining how individuals are distributed in the environment, what intervals along environmental gradients are selected or avoided, and what is most important to the species' growth and survival (Boesch \& Turner 1984, Baltz et al. 1998). For controlling variables, the central tendency of a distributional pattern is generally an indication of optimum or near-optimum conditions (Saucier \& Baltz 1993). Population density, survival, secondary production and individual growth should respond to gradients in habitat quality (Chatry et al. 1983, Baltz et al. 1998). In this study, we found that patterns of microhabitat use among and within species differed substantially and often statistically, and suggested strong resource partitioning (Ross 1986). Overlaps in resource use were generally low $(0=0.24$, range $=0.01$ to 0.59 ), especially between closely related species, where proportional overlap ranged from 0.19 to 0.38. Ecological segregation (Ross 1986) appeared to be general throughout the assemblage with the species assemblage structure changing along temporal, spatial and physico-chemical gradients in the factor analysis of microhabitat use (Fig. 5). These graphical models are useful to resource managers who want to know how assemblage structure will change if important environmental gradients (e.g. salinity and temperature) are modified (see also Walsh et al. 1999). For example, an increase of freshwater input into the basin would probably disfavor lesser blue crabs, offshore tonguefish and roughneck shrimp compared to blue crabs, blackcheek tonguefish and brown and white shrimp (Fig. 5A). Such knowledge of a species' environmental requirements can be used to sustain or enhance nursery functions for key estuarinedependent species (e.g. Chatry et al. 1983).

\section{Temperature}

Seasonal temperature changes, which are often cues for migrations and spawning (Gunter 1938, 1945, Lind- 
ner \& Anderson 1956, Miller 1965, Olney \& Grant 1976), were related to dynamic changes in assemblage structure. These dynamics were well illustrated by flatfishes. Our catches of large offshore and blackcheek tonguefishes were low in the summer and early fall indicating an offshore migration to avoid warm summer water temperatures (Gunter 1945, Miller 1965), but spawning from spring to summer (Olney \& Grant 1976) resulted in the appearance in Barataria Basin of new recruits in summer and fall. Bay whiff recruitment began in winter and peaked in spring (Gunter 1945, Gunter \& Hall 1963) and studies suggest that larger bay whiff migrate offshore in winter in response to cooling water temperatures (Gunter 1938, 1945). Similarly, in the southwestern region of the United Kingdom, the seasonal occurrence of juvenile sole Solea solea is controlled more by temperature than salinity (Claridge \& Potter 1987).

Seasonal conditions such as the passage of cold fronts in winter may mediate the recruitment of commercially important species, e.g. brown shrimp and blue crab (St. Amant et al. 1963, More 1969, Perry 1975, Perry \& Stuck 1982, Rogers et al. 1993). During frontal passage, northerly winds push the shallow bay water offshore, then when winds subside, warmer, more saline water from the Gulf refills the estuary (Chuang \& Wiseman 1983). Densities of brown shrimp in Barataria Basin increase from late March to midMay (Gaidry \& White 1973), then decrease primarily due to fishing mortality after the opening of the spring shrimp season in May, and our findings were consistent with that pattern (Fig. 4C). Blue crab, on the other hand, appeared to recruit to the estuary throughout the year reaching peak abundance in the winter (Fig. 4A). In Mississippi, the smallest crab stages are found in all seasons, indicating continual recruitment into the juvenile population (Perry 1975). Like brown shrimp, there is evidence of a strong relationship between movements of cold fronts and tides, and increased influx of blue crab megalopae from offshore (More 1969, Perry 1975, Perry \& Stuck 1982).

\section{Salinity}

Salinity differences played a relatively small role in resource use within species (Table 4), but among species it appeared to be more important. Species, including lesser blue crab, roughneck shrimp, offshore tonguefish and fringed flounder, were primarily found in the lower strata nearest the Gulf of Mexico. Some studies suggest that the lesser blue crab does not osmoregulate as well as the blue crab (Engel 1977, Piller et al. 1995), but Guerin \& Stickle (1997a) found that after long periods of exposure to low salinities
(2.5 psu), there is no difference in the hemolymph osmolality of juveniles of lesser blue and blue crabs. Nevertheless, lesser blue crabs do exhibit a decrease in growth and an increase in energy expenditure, especially for respiration and excretion, at low salinities (Guerin \& Stickle 1997a,b). Thus, higher salinities provide more suitable physiological conditions for lesser blue crab.

Barataria Basin's salinity and faunal gradients are far different from those typically found along the East Coast of the US (Thompson \& Forman 1987). The basin is shallow with relatively low salinity, no direct riverine inflow and a microtidal range. The freshwater portions of the basin begin about $30 \mathrm{~km}$ inland, which means offshore spawning species whose young migrate to freshwater nursery grounds must traverse long distances. Interannual variability in climate (Childers et al. 1990) also affects local habitat quality and accessibility and subsequently the distribution of estuarine-dependent organisms. For estuarine-dependent species, seasonal and interannual variation in environmental conditions (e.g. water surface elevation, temperature and salinity) may act as ecological filters (Wiens 1977, Beck 1995) that define the quantity of suitable habitat for early life history stages.

\section{Spatial scale, seasonality and ontogeny}

While large-scale variations in environmental conditions, e.g. temperature and salinity gradients, generally dominate resource use patterns of estuarinedependent species, small-scale changes are also important. The interface between Spartina marsh and open water has been confirmed by quantitative studies as an important nursery with large concentrations of juvenile fishes and macroinvertebrates (Zimmerman \& Minello 1984). Gametes spawned offshore are the source of many fish larvae that eventually move into the estuary and concentrate as juveniles near the marsh edge. While juvenile fishes are too small to be of interest to avian predators (Kneib 1982), they may use flooded Spartina or shallows along the water's edge to avoid larger piscivorous fishes. As these small fishes increase in size, they probably become less vulnerable to fish predators and more vulnerable to birds, and undergo a habitat shift from shallow marsh edges to deeper water (Baltz et al. 1993). In this study, 8 of the 13 selected species showed significant ontogenetic shifts toward deeper water as they grew (Table 4). In the present study, individuals of the smallest size class (SC1) were absent or scarce for brown and white shrimp, bay anchovy, spot and blackcheek tonguefish; however, Baltz et al. (1993) found that these fish species in SC1 were major components of the marsh-edge assemblage in the same study area. 
When seasonal and spatial patterns of estuarine use were analyzed together, a similar seasonal pattern of use was responsible for 2 of the 3 overlaps greater than 0.50 (Table 5). Species with peak abundance in the estuary during the same seasons had high overlaps ( $\geq 0.51)$. Closely related species, e.g. brown and white shrimps or blue and lesser blue crabs, that recruited to the estuary at different times of the year, had belowaverage overlaps $(\leq 0.26)$. Both Atlantic croaker and spot were most abundant in winter and spring (Fig. 2B,C) and Parker (1971) concluded from diet and distributional data that they were in direct competition; however, differences in size structure and overall abundance patterns in Barataria Basin probably accounted for their relatively low (0.38) overlap. This is evidence for some degree of resource partitioning (Ross 1986), but insufficient to demonstrate current competition.

Temporal resource partitioning (Ross 1986), especially in reproduction, is important in coastal marine communities. Spacing of the occurrence of larval fishes reduces potential trophic competition during the critical period (May 1974). Seasonal temperature changes also affect large-scale spatial distributions of juveniles (Day et al. 1989). At the microhabitat level, 12 of the 13 selected species that we analyzed showed size-related shifts in temperature use (Table 4), but, for some, the shifts may have been due to the ephemeral status of life history stages and changing seasonal conditions, making it difficult to unravel phenology into separate effects of seasonality and ontogeny (Miller 1979).

When plots of size classes in 3-dimensional environmental space (Fig. 5) were examined in conjunction with length-frequency distributions (Figs. 2, 3 \& 4), it was somewhat easier to interpret which species were showing effects of seasonality rather than ontogenetic shifts. For example, brown shrimp shifted to warmer, less oxygenated water with increasing size (Fig. 5C) probably because abundance, especially of the 2 largest size classes, was highest in spring and summer (Fig. 4C). Blue crab showed shifts in both directions, first to colder water with higher dissolved oxygen concentration then back toward warm, less oxygenated water (Fig. 5C), due to the higher abundances of smaller size classes (SC1 and SC2) in winter, while larger individuals were only collected in warmer seasons (Fig. 4A). Roughneck shrimp used decreasing temperature with increasing size (Fig. 5C) and were most abundant in winter; however, their absence from summer samples may indicate that after using the estuary from late fall to early spring they moved to cooler water offshore. Fishes with peak recruitment in winter and larger individuals present only in warmer months, e.g. Atlantic croaker, spot, bay whiff, and blackcheek tonguefish, showed similar shifts in temperature use that are probably more attributable to seasonally changing conditions than to ontogeny.

\section{Estuarine dependence}

In contrasting fish assemblages in western Australia and southern Africa, Potter et al. (1990) took an anthropocentric view of fish utilization of coastal habitat types, particularly with regard to estuarine dependence. They adopted a geological definition of an estuary (Pritchard 1967) and then modified it to also include water bodies that are only periodically open to the sea. Even if the existence of a given estuary is ephemeral on a geological timescale, estuarine-like conditions (e.g. reduced salinity, high turbidity and shallow depths) probably have existed consistently enough in most regions to allow fishes that prefer them to persist. Thus, it seems inappropriate to refute the concept of estuarine dependence for life history stages of species found in estuarine-like conditions in systems that do not meet Pritchard's modified definition of an estuary (Pritchard 1967). We prefer to take a fish's eye view of habitat (Chesney et al. 2000) and use the distribution and abundance of a species or life history stage over environmental gradients to define its habitat. Taking this view, a species may be characterized as estuarine-dependent if a life history stage requires some combination of environmental conditions typically found in estuaries. Environmental conditions that control recruitment success for a species may vary across its range (Miller 1994), so a species may be dependent on estuaries (low salinity shallows, rich in prey) throughout most of its range but rely heavily on seagrass-covered coastal sand flats (low salinity shallows, rich in prey) in another location. As Potter et al. (1990) suggested (p. 168), the identification of nursery habitat types should require at least a comparison of the densities of early life history stages in other nearby habitat types (Pearcy \& Myers 1974, Walsh et al. 1999), but a better characterization would derive from enhanced survival, growth and recruitment (Boesch \& Turner 1984). Thus, the occurrence of a life history stage in more than 1 habitat type is insufficient to refute the nursery function of a given habitat type. A species may be estuarine-dependent on one end of its range and rely on similar, estuarine-like conditions at another extreme; it is therefore the controlling variables that are important and not the biologist's definition and characterization of a habitat type.

\section{Turbidity}

Although not measured in this study, we feel that turbidity is extremely important (Chesney et al. 2000). However, in a related study (Baltz et al. 1993) turbidity only loaded heavily ( $>0.50$ ) on the 4 th principal component; it is not a major gradient of environ- 
mental variation in Louisiana's highly turbid systems. The role of turbidity may be the least appreciated feature contributing to the high productivity of Louisiana's coastal fisheries, but it enhances the nursery function of the estuaries, probably by enhancing the survival of early life history stages. Blaber \& Blaber (1980) identified the high turbidity of estuarine waters as a controlling variable for juveniles of many estuarine-dependent fishes, and linked high turbidity to reduced predation pressure and high concentrations of food associated with shallow waters. Cyrus \& Blaber $(1987 a, b)$ examined 10 South African fishes in field and laboratory studies of turbidity use and preference and found that 6 of 10 marine fishes exhibited preferences in laboratory experiments that were generally concordant with field selection data. Cyrus \& Blaber (1987c) also found that the distributions of 8 of 20 juvenile fishes were correlated with patterns of their favored prey in the benthos and suggested that turbid waters provide a form of cover for fishes by reducing the reactive distance of their predators. Marshall \& Elliott (1998) lumped all individuals of a species together, so the validity of their assertion that turbidity is unimportant in the Humber Estuary, UK, as a variable structuring the fish assemblage is difficult to assess without an evaluation of its influence across size classes (Livingston 1988).

Understanding the patterns of resource use and the environmental requirements of estuarine-dependent species is an important step in identifying and protecting nursery habitats. We used a microhabitat approach to examine patterns of resource use (Ross 1986) across complex gradients in a large estuarine system. This approach demonstrated a high degree of ecological segregation among and within species and offered insights into the changing environmental requirements (Livingston 1988) of important species and life history stages. Identifying patterns of microhabitat utilization by season and size class can be used to develop knowledge of species' responses to controlling environmental variables for important life history functions (Saucier \& Baltz 1993, Baltz et al. 1998). Microhabitat data developed for populations can also be extended to analyses at the community level. The factor analysis approach that we used offers insights into community management issues and can be used to predict how changing environmental conditions will influence assemblage structure. Factor analysis simplifies complex, multiple-variable gradients, places species in the context of their community and shows how assemblage structure will respond by allowing resource managers to identify species or life history stages that will be favored or disfavored by environmental impacts, water management options or habitat restorations.
Acknowledgements. We are grateful to M. Reichert, D. Nieland, J. Buchanan, D. MacNaughton, K. Duffy, V. Hunt, J. Bearden, P. Hoffman, C. Wilson, J. Render, F. Delatte, L. Lynch, A. Stokes, T. Schaefer, W. Lamb, C. Lamb, B. Himel and $\mathrm{S}$. Lane for assisting with the field work. We also appreciate comments by E. Chesney, B. Marx, L. Rouse and 3 anonymous reviewers on the manuscript. A. Arrivillaga provided valuable help with invertebrate identification and statistical analyses. B. Thompson provided help with fish identification. Finally, we gratefully acknowledge the field support of the Louisiana Department of Wildlife and Fisheries personnel, especially Claude Boudreaux, at the Lyle S. St. Amant Marine Biological Laboratory on Grand Terre, Louisiana.

\section{LITERATURE CITED}

Allen RL, Baltz DM (1997) Distribution and microhabitat use by flatfishes in a Louisiana estuary. Environ Biol Fishes 50: 85-103

Baltz DM (1990) Autecology. In: Schreck CB, Moyle PB (eds) Methods for fish biology. American Fisheries Society, Bethesda, MD, p 583-605

Baltz DM, Rakocinski CF, Fleeger JW (1993) Microhabitat use by marsh-edge fishes in a Louisiana estuary. Environ Biol Fishes 36:109-126

Baltz DM, Fleeger JW, Rakocinski CF, McCall JN (1998) Food, density, and microhabitat: factors affecting growth and recruitment potential of juvenile saltmarsh fishes. Environ Biol Fishes 53:89-103

Baumann RH (1987) Physical variables. In: Conner WH, Day JW Jr (eds) The ecology of Barataria Basin, Louisiana: an estuarine profile. US Fish Wildl Serv Biol Rep 85(7.13): 8-17

Beck MW (1995) Size-specific shelter limitation in stone crabs: a test of the demographic bottleneck hypothesis. Ecology 76:968-980

Blaber SJM, Blaber TG (1980) Factors affecting the distribution of juvenile estuarine and inshore fish. J Fish Biol 17: 143-162

Boesch DF, Turner RE (1984) Dependence of fishery species on salt marshes: the role of food and refuge. Estuaries 7: $460-468$

Chatry M, Dugas RJ, Easley KA (1983) Optimum salinity regime for oyster production on Louisiana's state seed grounds. Contrib Mar Sci 26:81-94

Chesney EJ, Baltz DM (2001) The effects of hypoxia on the northern Gulf of Mexico coastal ecosystem: A fisheries perspective. In: Rabalais NN, Turner RE (eds) Coastal hypoxia: consequences for living resources and ecosystems. Coastal \& estuarine studies 58. American Geophysical Union, Washington, DC, p 321-354

Chesney EJ, Baltz DM, Thomas RG (2000) Louisiana estuarine and coastal fisheries and habitats: perspectives from a fish's eye view. Ecol Appl 10:350-366

Childers DL, Day JW Jr, Muller RA (1990) Relating climatological forcing to coastal water levels in Louisiana estuaries and the potential importance of El Niño-Southern Oscillation events. Clim Res 1:31-42

Chuang WS, Wiseman WJ Jr (1983) Coastal sea level response to frontal passages on Louisiana-Texas shelf. J Geophys Res 91:10545-10659

Claridge PN, Potter IC (1987) Size composition and seasonal changes in abundance of juvenile sole, Solea solea, in the Severn Estuary and inner Bristol Channel. J Mar Biol Assoc UK 67:561-569

Conner WH, Day JW Jr (1987) Description of the basin. In: 
Conner WH, Day JW Jr (eds) The ecology of Barataria Basin, Louisiana: an estuarine profile. US Fish Wildl Ser Biol Rep 85(7.13):1-7

Conner WH, Day JW Jr, Gosselink JG, Hopkinson CS Jr, Stowe WC (1987) Vegetation: composition and production. In: Conner WH, Day JW Jr (eds) The ecology of Barataria Basin, Louisiana: an estuarine profile. US Fish Wildl Ser Biol Rep 85(7.13):31-47

Cushing DH (1973) Recruitment and parent stock in fishes. University of Washington Press, Seattle

Cyrus DP, Blaber SJM (1987a) The influence of turbidity on juvenile marine fishes in estuaries. Part 1 . Field studies at Lake St. Lucia on the southeastern coast of Africa. J Exp Mar Biol Ecol 109:53-70

Cyrus DP, Blaber SJM (1987b) The influence of turbidity on juvenile marine fishes in estuaries. Part 2. Laboratory studies, comparisons with field data and conclusions. J Exp Mar Biol Ecol 109:71-91

Cyrus DP, Blaber SJM (1987c) The influence of turbidity on juvenile marine fishes in estuaries of Natal, South Africa. Cont Shelf Res 7:1411-1416

Day JW Jr, Hall CAS, Kemp WM, Yanez-Arancibia A (1989) Estuarine ecology. Wiley-Liss, New York

Engel DW (1977) Comparison of the osmoregulatory capabilities of two portunid crabs, Callinectes sapidus and C. similis. Mar Biol 41:275-279

Fahay MP (1983) Guide to the early stages of marine fishes occurring in the western North Atlantic Ocean, Cape Hatteras to the Southern Scotian Shelf. J NW Atl Fish Sci $4: 1-423$

Gaidry WJ III, White CJ (1973) Investigations of commercially important penaeid shrimp in Louisiana estuaries. Louisiana Wildl Fish Comm Tech Bull No 8

Guerin JL, Stickle WB (1997a) A comparative study of two sympatric species within the genus Callinectes: osmoregulation, long-term acclimation to salinity and the effects of salinity on growth and moulting. J Exp Mar Biol Ecol 218: 165-186

Guerin JL, Stickle WB (1997b) Effect of salinity on survival and bioenergetics of juvenile lesser blue crabs, Callinectes similis. Mar Biol 129:63-69

Gunter G (1938) Seasonal variations in abundance of certain estuarine and marine fishes in Louisiana with particular reference to life histories. Ecol Monogr 8:314-346

Gunter G (1945) Studies on marine fishes of Texas. Publ Inst Mar Sci Univ Tex 1:1-190

Gunter G, Hall GE (1963) Biological investigations of the St. Lucie estuary (Florida) in connection with Lake Okeechokee discharges through the St. Lucie canal. Gulf Res Rep 1:189-307

Hjort J (1914) Fluctuations in the great fisheries of northern Europe viewed in the light of biological research. Rapp P-V Reun Cons Int Explor Mer 20:1-228

Hoese HD, Moore RH (1977) Fishes of the Gulf of Mexico. Texas, Louisiana and adjacent waters. Texas A\&M Univ Press, College Station

Hopkins TS, Valentine JF, Lutz LB (1989) An illustrated guide with key to selected benthic invertebrate fauna of the northern Gulf of Mexico. Dauphin Island Sea Laboratory, Dauphin Island, Alabama

Houde ED (1987) Fish early life dynamics and recruitment variability. Am Fish Soc Symp 2:17-29

Houde ED (1989) Subtleties and episodes in the early life of fishes. J Fish Biol 35(Suppl A):29-38

Houde ED, Rutherford ES (1993) Recent trends in estuarine fisheries: Predictions of fish production and yield. Estuaries 16:161-176
Hurlbert SH (1981) A gentile depilation of the niche: Dicean resource sets in resource hyperspace. Evol Theor 5: $177-184$

Hurlbert SH (1984) Pseudoreplication and the design of ecological field experiments. Ecol Monogr 54:187-211

Jobling M (1995) Environmental biology of fishes. Chapman \& Hall, New York

Kneib RT (1982) The effects of predation by wading birds (Ardeidae) and blue crabs (Callinectes sapidus) on the population structure of the common mummichog, Fundulus heteroclitus. Estuar Coast Shelf Sci 14:159-165

Kuipers B (1975) On the efficiency of a two-meter beam trawl for juvenile plaice (Pleuronectes platessa). Neth J Sea Res 9:69-85

Lindner MJ, Anderson WW (1956) Growth, migrations, spawning and size distribution of shrimp Penaeus setiferus. US Fish Wildl Serv Fish Bull 56:554-645

Livingston RJ (1988) Inadequacy of species-level designations for ecological studies of coastal migratory fishes. Environ Biol Fishes 22:225-234

Magnuson JJ, Crowder LB, Medvick PA (1979) Temperature as an ecological resource. Am Zool 19:331-343

Marshall S, Elliott M (1998) Environmental influences of the fish assemblage of the Humber Estuary, UK. Estuar Coast Shelf Sci 46:175-184

Martin F, Drewry GE (1978) Development of fishes of the midAtlantic bight: an atlas of eggs, larval, and juvenile stages. Vol. 6. Stromateidae through Ogcocephalidae. US Fish Wildl Serv, Biol Serv Prog FWS/OBS-78/12

May RC (1974) Larval mortality in marine fishes and the critical period concept. In: Blaxter JHS (ed) The early life history of fish. Springer-Verlag, New York, p 3-19

Miller JM (1965) A trawl survey of the shallow Gulf fishes near Port Aransas, Texas. Publ Inst Mar Sci Univ Tex 10: 80-107

Miller JM (1994) An overview of the second flatfish symposium: Recruitment in flatfish. Neth J Sea Res 32:103-106

Miller JM, Burke JS, Fitzhugh GR (1991) Early life history patterns of Atlantic North American flatfish: likely (and unlikely) factors controlling recruitment. Neth J Sea Res 27:261-275

Miller PJ (1979) A concept of fish phenology. Symp Zool Soc Lond 44:1-28

Miller TJ, Crowder LB, Rice JA, Marschall EA (1988) Larval size and recruitment mechanisms in fishes: Towards a conceptual framework. Can J Fish Aquat Sci 45: 1657-1670

Minello TJ (1999) Nekton densities in shallow estuarine habitats of Texas and Louisiana and the identification of essential fish habitat. In: Beneka RL (ed) Fish habitat: essential fish habitat and rehabilitation. Am Fish Soc Symp 22: 43-75

Minello TJ, Zimmerman RJ, Martinez EX (1989) Mortality of young brown shrimp Penaeus aztecus in estuarine nurseries. Trans Am Fish Soc 118:693-708

More WR (1969) A contribution to the biology of the blue crab (Callinectes sapidus Rathbun) in Texas with a description of the fishery. Texas Parks Wildl Dep Tech Ser 1:1-31

Munroe TA (1991) Western Atlantic tonguefishes of the Symphurus plagusia complex (Cynoglossidae: Pleuronectiformes), with descriptions of two new species. Fish Bull US 89:247-287

Munroe TA, Allen RL, Baltz DM, Ross SW (2000) Symphurus civitatium (Pleuronectifromes: Cynoglossidae), a second estuarine-occurring tonguefish off the southeastern United States and northern Gulf of Mexico. Estuaries 23: 439-448 
Olney JE, Grant GC (1976) Early planktonic larvae of the blackcheek tonguefish, Symphurus plagiusa (Pisces: Cynoglossidae), in the lower Chesapeake Bay. Chesapeake Sci 17:229-237

Parker JC (1971) The biology of spot, Leiostomus xanthurus Lacepede, and Atlantic croaker, Micropogon undulatus (Linnaeus), in two Gulf of Mexico nursery areas. Sea Grant Publ No TAMU-SG-71-210. Texas A\&M Univ, College Station. Available at: http://nsgl.gso.uri.edu/tamu/ tamut71004.pdf

Pearcy WG, Myers SS (1974) Larval fishes of Yaquina Bay, Oregon: a nursery ground for marine fishes? Fish Bull US 72:201-213

Perry HM (1975) The blue crab fishery in Mississippi. Gulf Res Rep 5:39-57

Perry HM, Stuck KC (1982) The life history of the blue crab in Mississippi with notes on larval distribution. In: Perry HM, Van Engel WA (eds) Proc Blue Crab Colloq, Oct 16-18, 1979, Biloxi, Mississippi, Vol 7. Gulf States Marine Fisheries Commission, Ocean Springs, MS, p 17-22

Piller SC, Henry RP, Doeller JE, Kraus DW (1995) A comparison of the gill physiology of two euryhaline crab species, Callinectes sapidus and Callinectes similis. I. Energy production, transport-related enzymes and osmoregulation as a function of acclimation salinity. J Exp Biol 198: 349-358

Potter IC, Beckley LE, Whitfield AK, Lenanton RCJ (1990) Comparison between the roles played by estuaries in the life cycles of fishes in temperate western Australia and Southern Africa. Environ Biol Fishes 28:143-178

Pritchard DW (1967) What is an estuary: physical viewpoint. In: Lauff GH (ed) Estuaries. Publication No. 53. American Association for the Advancement of Science, Washington, DC, $\mathrm{p} 3-5$

Rogers BD, Shaw RF, Herke WH, Blanchet RH (1993) Recruitment of postlarval and juvenile brown shrimp (Penaeus aztecus Ives) from offshore to estuarine waters of the northwestern Gulf of Mexico. Estuar Coast Shelf Sci 36: 377-394

Rose KA, Cowan JH Jr, Houde ED, Coutant CC (1993) Indi-

Editorial responsibility: Ronald Kneib (Contributing Editor), Sapelo Island, Georgia, USA vidual-based modelling of environmental quality effects on early life stages of fishes: a case study using striped bass. Am Fish Soc Symp 14:125-145

Ross ST (1986) Resource partitioning in fish assemblages: a review of field studies. Copeia 1986:352-388

SAS Institute (1996) SAS/STAT guide for personal computers. Version 6 edn. SAS Institute, Cary, NC

Saucier MH, Baltz DM (1993) Spawning site selection by spotted seatrout, Cynoscion nebulosus, and black drum, Pogonias cromis, in Louisiana. Environ Biol Fishes 36:257-272

Schoener TW (1970) Non-synchronous spatial overlap in lizards in patchy habitats. Ecology 51:408-418

St. Amant LS, Corkum KC, Broom JG (1963) Studies on growth dynamics of brown shrimp, Penaeus aztecus, in Louisiana waters. Proc Gulf Caribb Fish Inst 15:14-26

Thompson BA, Forman W (1987) Nekton. In: Conner WH, Day JW Jr (eds) The ecology of Barataria Basin, Louisiana: an estuarine profile, US Fish Wildl Ser Biol Rep 85(7.13): 80-95

Turner RE (1997) Wetland loss in the northern Gulf of Mexico: Multiple working hypotheses. Estuaries 20:1-13

Walsh HJ, Peters DS, Cyrus DP (1999) Habitat utilization by small flatfishes in a North Carolina estuary. Estuaries 22: 803-813

Walters CJ, Collie JS (1988) Is research on environmental factors useful to fisheries management? Can J Fish Aquat Sci 45:1848-1854

Wiens JA (1977) On competition and variable environments. Am Sci 65:590-597

Williams AB (1984) Shrimps, lobsters, and crabs of the Atlantic Coast of the eastern United States, Maine to Florida. Smithsonian Institution Press, Washington, DC

Zimmerman RJ, Minello T (1984) Densities of Penaeus aztecus, Penaeus setiferus, and other natant macrofauna in a Texas salt marsh. Estuaries 7:421-433

Zimmerman RJ, Minello TJ, Rozas LP (2000) Salt marsh linkages to productivity of penaeid shrimps and blue crabs in the northern Gulf of Mexico. In: Weinstein MP, Kreeger DA (eds) Concepts and controversies in tidal marsh ecology. Kluwer Academic Publishers, Dordrecht, p 293-314

Submitted: July 5, 2000; Accepted: January 23, 2002

Proofs received from author(s): June 7, 2002 